\title{
THE EQUIVARIANT HUREWICZ MAP
}

\author{
L. GAUNCE LEWIS, JR.
}

\begin{abstract}
Let $G$ be a compact Lie group, $Y$ be a based $G$-space, and $V$ be a $G$-representation. If $\pi_{V}^{G}(Y)$ is the equivariant homotopy group of $Y$ in dimension $V$ and $H_{V}^{G}(Y)$ is the equivariant ordinary homology group of $Y$ with Burnside ring coefficients in dimension $V$, then there is an equivariant Hurewicz map

$$
h: \pi_{V}^{G}(Y) \rightarrow H_{V}^{G}(Y) .
$$

One should not expect this map to be an isomorphism, since $H_{V}^{G}(Y)$ must be a module over the Burnside ring, but $\pi_{V}^{G}(Y)$ need not be. However, here it is shown that, under the obvious connectivity conditions on $Y$, this map induces an isomorphism between $H_{V}^{G}(Y)$ and an algebraically defined modification of $\pi_{V}^{G}(Y)$.

The equivariant Freudenthal Suspension Theorem contains a technical hypothesis that has no nonequivariant analog. Our results shed some light on the behavior of the suspension map when this rather undesirable technical hypothesis is not satisfied.
\end{abstract}

\section{INTRODUCTION}

Let $G$ be a compact Lie group and $Y$ be a based $G$-space. One obvious way to form an equivariant analog of the homotopy groups of a space is to assign to each $G$-representation $V$ the set $\pi_{V}^{G}(Y)$ of based $G$-homotopy classes of $G$ maps from the one-point compactification $S^{V}$ of $V$ to $Y[4,7,8,21]$. These equivariant homotopy sets are certainly of interest in the study of $G$-spaces, but they are rather hard to compute. Perhaps the most basic tools available for analyzing the nonequivariant homotopy groups of spaces are the Hurewicz map, the associated isomorphism theorem, and Whitehead's extension of that theorem to an epimorphism result in the next higher dimension [23]. To obtain an equivariant analog of these tools, one must first define the homology of a $G$ space $Y$ in dimension $V$. By introducing $\mathrm{RO}(G)$-graded equivariant ordinary cohomology and homology in [14], May, McClure, and the author have provided this definition. In this paper, the equivariant Hurewicz map

$$
h: \pi_{V}^{G}(Y) \rightarrow H_{V}^{G}(Y)
$$

Received by the editors November 20, 1989.

1991 Mathematics Subject Classification. Primary 54H15, 55N91, 55P42, 55P91, 55Q10, 55Q91; Secondary 55M35, 55N10, 57S15.

Key words and phrases. Hurewicz map, Hurewicz isomorphism, Whitehead theorem, equivariant ordinary homology, Freudenthal Suspension Theorem.

Supported in part by National Science Foundation Grant MCS-8301336. 
is defined and, for representations $V$ with the real dimension of $V^{G}$ greater than or equal to 2, analogs of the Hurewicz Isomorphism Theorem and Whitehead's extension are proved.

Since $\mathrm{RO}(G)$-graded equivariant homology is defined by producing a representing spectrum, the natural route to these results is to first prove a stable Hurewicz theorem and then derive the unstable results via an equivariant Freudenthal Suspension Theorem. Our work on the stable Hurewicz theorem (Theorem 2.1) is contained in $\S 2$. This theorem is an example of those pleasant equivariant results which seem to be proved simply by inserting $G$ 's in all the right places. The details provided are only those necessary to accomplish two goals. The first is to make clear the reason for our choice of the Burnside ring as the coefficients for our homology groups. It seems that the introduction to $G$-spectra given in the first two chapters of [15] is more demanding than had been intended. Thus, the second goal is to provide a simple example of an application of this material.

The second step in our approach to the unstable equivariant Hurewicz theorem, that of deriving unstable results from stable ones, is an example of exactly the opposite sort of result in equivariant homotopy theory. All sorts of difficulties arise which one might not expect based on the nonequivariant case. Fundamentally, the problem here is simply that the gap between stable and unstable homotopy theory in the equivariant context is vastly wider than it is in the nonequivariant context. The most obvious sign of this is the fact that, in the nonequivariant context, the unstable homotopy groups $\pi_{n}(X)$ and the homology groups $H_{n}(X)$ of a space $X$ are the same sort of objects-that is, abelian groups-as long as $n \geq 2$. In the equivariant context, the homology groups $H_{V}^{G}(Y)$ of a $G$-space $Y$ always carry the structure of Mackey functors, while for $G$ compact and $Y$ not highly connected, the $G$-homotopy groups $\pi_{V}^{G}(Y)$ need not have such a structure in any dimension $V$. Of course, by imposing extremely strict connectivity conditions on $Y$ and choosing $V$ very carefully, one may ensure that the group $\pi_{V}^{G}(Y)$ carries the structure of a Mackey functor. Under such very strict conditions, one obtains exact analogs of the nonequivariant Hurewicz and Whitehead results (Theorem 1.3). As indicated in Remarks 2.2 , these results provide some information about the equivariant homotopy groups of spheres. Nevertheless, the required conditions on $Y$ and $V$ are simply too strict for most applications.

The increased gap between stable and unstable homotopy theory is reflected in the equivariant Freudenthal Suspension Theorem by the presence of an unexpected restriction (conditions (b) of Theorem 3.3) on the relations among the dimensions of the fixed point sets $V^{K}$ of $V$ for certain subgroups $K$ of $G$ and the connectivity of the fixed point subsets $Y^{J}$ of $Y$ with respect to certain subgroups $J$ of the subgroups $K$. As we explain in $\S 3$, these relations may be regarded as gap conditions on the dimensions of the fixed point sets of $V$. The primary purpose of this paper is to show that, at least in the dimensions appropriate to the Hurewicz Isomorphism Theorem, one can very precisely describe the consequences of the failure of $V$ to satisfy these gap hypotheses. If $V$ does not satisfy them, then the Hurewicz map

$$
h: \pi_{V}^{G}(Y) \rightarrow H_{V}^{G}(Y)
$$

need not be an isomorphism. However, even in the nonequivariant context, we 
encounter a dimension, namely $n=1$, in which the Hurewicz map

$$
h: \pi_{n}(X) \rightarrow H_{n}(X)
$$

need not be an isomorphism. We all know what to do; by abelianizing $\pi_{1}(X)$, we obtain an isomorphism. Our main results, Theorems 1.7 and 1.8 , have a similar form; that is, if the dimension of $V^{G}$ is greater than or equal to 2 and $Y$ is $G-(V-1)$-connected, then

$$
h: \pi_{V}^{G}(Y) \rightarrow H_{V}^{G}(Y)
$$

becomes an isomorphism after $\pi_{V}^{G}(Y)$ is altered in an appropriate way. Our results have, however, one very big advantage over the nonequivariant result on $\pi_{1}(X)$. The existence of nontrivial perfect groups implies that $H_{1}(X)$ can vanish when $\pi_{1}(X)$ does not. No such undetectable homotopy groups occur in our context.

Previous versions of the equivariant Freudenthal Suspension Theorem have imposed unexpected and unwelcome extra hypotheses to obtain the result that the suspension map is an isomorphism. Two of our intermediate results, Theorem 5.1 and Proposition 6.1, may be regarded as suspension theorems of a more sophisticated sort. Instead of imposing these extra conditions, they explain precisely how the domain of the suspension map $\sigma$ must be modified so that $\sigma$ becomes an isomorphism. Even though these results apply only to a rather limited range of dimensions, they should be of independent interest. Proposition 6.1 should be of special interest since it sheds a bit of light on the behavior of the change of universe functors [15, §II.1] which appear ubiquitously in equivariant stable homotopy theory.

Two other results should be of independent interest. One, Theorem 1.11, is an equivariant version of Whitehead's result relating the behavior in homology and homotopy of a map $f: Y \rightarrow Z$. The other, Lemma 1.2, relates two measures of the connectivity of a $G$-space $Y$. One measure is in terms of the connectivity of the various fixed point subspaces of $Y$. The other is in terms of the vanishing of certain homotopy groups $\pi_{W}^{H}(Y)$, where $K$ ranges over the subgroups of $G$ and $W$ over certain nontrivial $K$-representations. Much of the force of the equivariant Hurewicz theorem should come out of the connection between these two measures of connectivity.

A few remarks are appropriate about the relation of the results here to previous work. Our debt to Waner [21] is deep. He proves, for finite groups, a Whitehead theorem like Theorem 1.11, a Hurewicz theorem like Theorem 1.3, and Lemma 1.2. Waner uses rather sophisticated geometric techniques, based on his notion of a $V$-CW complex for a representation $V$, to prove his results. Our search for homotopy-theoretic variants of his tools led us to the proof of Theorem 5.1. Hauschild proves a Hurewicz Isomorphism Theorem [7], but for an equivariant homology theory that does not satisfy the dimension axiom. Also, he was aware of some form of Lemma 1.2. Lück [16] proves an equivariant Hurewicz Isomorphism Theorem, but his result is of an essentially different nature. It deals with the homotopy and homology groups of the various fixed point subspaces of a $G$-space $Y$, assembled into functors designed for the study of non-simply-connected $G$-spaces.

This paper is organized so that all the basic definitions and the main results on unstable homotopy groups appear in $\S 1$. All the work on the stable Hurewicz 
map and theorem is in $\S 2$. Our simplest unstable Hurewicz theorem is derived in $\S 3$ from Namboodiri's equivariant Freudenthal Suspension Theorem. Various other technical results related to the suspension map also appear there. $\S 4$ is devoted to the functors which modify the homotopy groups $\pi_{V}^{G}(Y)$ so that they become isomorphic to the corresponding homology groups. One of our delicate equivariant suspension theorems, describing the extent to which the suspension map fails to be an isomorphism, is stated and proved in $\S 5$. A second such theorem, describing the behavior in low dimensions of the change of universe functors of [15], is given in $\S 6$. Also a preliminary version of our more sophisticated unstable Hurewicz theorem is stated and proved there. $\S 7$ is devoted to proving the connection between our two measures of equivariant connectivity. The absolute version of our stronger unstable Hurewicz theorem is proved there using this connection. The final section is devoted to the proofs of the relative unstable Hurewicz theorem and Whitehead's theorem.

This paper is, of course, based on the approach to equivariant stable homotopy theory developed in [15]. However, potential readers should be comforted to know that extensive knowledge of [15] is really not demanded. We employ the properties of $G-\mathrm{CW}$ spectra developed in [15, Chapter 1]. The statements of these properties are merely the obvious simultaneous generalization of the properties of $G-\mathrm{CW}$ complexes and nonequivariant $\mathrm{CW}$ spectra. Minimal use is made of the change of universe functors from [15, §II.1]. The generalization of Wirthmüller's isomorphism described in [15, §II.6] is used extensively, but anyone interested in equivariant homotopy theory and unfamiliar with his result will find it well worth learning. The basic definition of the transfer for bundles of the form $G / H \rightarrow G / K$ is used, together with the double coset formula for such bundles and one other nontrivial property from [15, §II.5]. Finally, basic information from $[15, \S \mathrm{V} .9]$ about the Burnside category is used. This category is an obvious generalization of the Burnside ring; the results used are all extensions of familiar facts about the Burnside ring.

\section{The UNSTABle HuREwicz ISOMORPHisM}

We begin by reviewing some basic concepts of equivariant homotopy theory in order to fix our notation. By group, we mean a compact Lie group; by subgroup, we mean a closed subgroup. The notation $K \leq G$ indicates that $K$ is a subgroup of the group $G$. All $G$-spaces are left $G$-spaces. Whenever possible, the prefix $G$ is omitted from our notations, so that by spaces, subspaces, maps, etc., we mean $G$-spaces, $G$-subspaces, $G$-maps, etc. If $K$ is a subgroup of $G$ and $Y$ is a $G$-space, then $Y^{K}$ is the $K$-fixed subspace of $Y$. A based $G$-space is a $G$-space $Y$ together with a specified basepoint, which is required to be in $Y^{G}$. If $X$ is any $G$-space, then $X^{+}$denotes the disjoint union of $X$ and a $G$-trivial basepoint. For based $G$-spaces $X$ and $Y,[X, Y]_{G}$ denotes the set of based $G$-homotopy classes of based $G$-maps from $X$ to $Y$.

All $G$-representations are assumed to have a $G$-invariant inner product. If $V$ is a $G$-representation, then $D V, S V$, and $S^{V}$ denote its unit disk, its unit sphere, and its one-point compactification, respectively. The basepoint of $S^{V}$ is the point at infinity. The dimension of $V$ over the real numbers is denoted $|V|$; similarly, the real virtual dimension of a virtual representation $\alpha$ is denoted $|\alpha|$. For each nonnegative integer $n$, the trivial $G$ representation of real 
dimension $n$ is denoted by $n$. If $V$ and $W$ are two $G$-representations, then $V+W$ denotes their direct sum. The orthogonal complement of a subrepresentation $W$ of a representation $V$ is denoted by $V-W$. Notice that, in the absence of an explicit remark to the contrary, $V-W$ in this article denotes an actual representation rather than a virtual representation. The direct sum of $m$ copies of $V$ is denoted $m V$. If $V$ is a $G$-representation and $Y$ is a based $G$-space, then the set $\left[S^{V}, Y\right]_{G}$ is denoted $\pi_{V}^{G}(Y)$ and called the $V$ th homotopy group (or set) of $Y$. Recall that, if $K \leq G$ and $n \geq 0$, then

$$
\pi_{n}^{K}(Y)=\left[S^{n}, Y\right]_{K} \cong\left[G / K^{+} \wedge S^{n}, Y\right]_{G} \cong \pi_{n}\left(Y^{K}\right) .
$$

Let $i: B \rightarrow Y$ be the inclusion of a $G$-subspace $B$ of $Y$ and let $F i$ be the homotopy fiber of $i$. For any $G$-representation $V$ containing at least one copy of the trivial representation, the relative homotopy set $\pi_{V}^{G}(Y, B)$ is defined to be $\pi_{V-1}^{G}(F i)$. The set $\pi_{V}^{G}(Y, B)$ may be identified with the set

$$
[(D V, S V, *),(Y, B, *)]_{G}
$$

of $G$-homotopy classes of $G$-maps of triples.

If $Y$ is a $G$-space, then $H_{*}^{G}(Y)$ denotes the reduced, $\operatorname{RO}(G)$-graded equivariant ordinary homology of $Y$ with Burnside ring coefficients. If $B$ is a $G$-subspace of $Y$, then $H_{*}^{G}(Y, B)$ denotes the $\mathrm{RO}(G)$-graded equivariant ordinary homology of the pair $(Y, B)$ with Burnside ring coefficients. For any $G$-representation $V$, there are natural Hurewicz maps

$$
h: \pi_{V}^{G}(Y) \rightarrow H_{V}^{G}(Y) \text { and } h: \pi_{V}^{G}(Y, B) \rightarrow H_{V}^{G}(Y, B) .
$$

In $\S 3$, we describe these maps as the composite of a stable Hurewicz map and the stabilization map from the unstable $V$ th homotopy group of $Y$ (or the pair $(Y, B))$ to the stable $V$ th homotopy group of $Y$ (or $(Y, B))$.

There are two ways to measure the equivariant connectivity of a $G$-space $Y$. The most common measure defines the equivariant connectivity of $Y$ in terms of the nonequivariant connectivity of the fixed subspaces $Y^{K}$ for the various subgroups $K$ of $Y$. A more general measure of the equivariant connectivity of $Y$ can be defined by considering the subgroups $K$ of $G$ and the $K$-representations $W$ for which $\pi_{W}^{K}(Y)$ is zero. Waner has described the relation between these two measures when the group $G$ is finite [21]. Here, we recall both measures and extend Waner's results on the relation between them to compact Lie groups. This relationship is the source of much of the power of the equivariant Hurewicz Isomorphism Theorem.

Definition 1.1. (i) A dimension function $n^{*}$ is a function from the set of conjugacy classes of subgroups of $G$ to the integers. The value of $n^{*}$ on the conjugacy class of $K \leq G$ is denoted $n^{K}$. If $n^{*}$ and $m^{*}$ are two dimension functions, then $n^{*} \geq m^{*}$ if $n^{K} \geq m^{K}$ for every subgroup $K$. Associated to any $G$-representation $V$ is the dimension function $\left|V^{*}\right|$ whose value at $K$ is the real dimension of the $K$-fixed subspace $V^{K}$ of $V$. The dimension function with constant value 2 is denoted $2^{*}$, and similarly for any other integer.

(ii) Let $n^{*}$ be a nonnegative dimension function. Then a $G$-space $Y$ is $G$ - $n^{*}$-connected if, for each subgroup $K$ of $G$, the fixed point space $Y^{K}$ is $n^{K}$-connected. A $G$-space $Y$ is $G$-connected if it is $G-0^{*}$-connected. Also, a 
$G$-space is simply $G$-connected if it is $G$ - $1^{*}$-connected. A space $Y$ is homologically $G-n^{*}$-connected if, for every subgroup $K$ of $G$ and every integer $m$ with $0 \leq m \leq n^{K}$, the homology group $H_{m}^{K}(Y)$ is zero.

(iii) Let $n^{*}$ be a nonnegative dimension function and let $f: Y \rightarrow Z$ be a $G$-map. Then $f$ is a $G-n^{*}$-equivalence if, for every subgroup $K$ of $G$,

$$
\left(f^{K}\right)_{*}: \pi_{m}\left(Y^{K}\right) \rightarrow \pi_{m}\left(Z^{K}\right)
$$

is an isomorphism for every integer $m$ with $0 \leq m<n^{K}$ and an epimorphism for $m=n^{K}$. A $G$-pair $(Y, B)$ is said to be $G-n^{*}$-connected if the inclusion of $B$ into $Y$ is a $G-n^{*}$-equivalence. The notions of homology $G-n^{*}$-equivalence and of homology $G-n^{*}$-connectedness for pairs are defined similarly, but with homotopy groups replaced by homology groups.

(iv) Let $V$ be a $G$-representation. For each subgroup $K$ of $G$, let $V(K)$ be the orthogonal complement of $V^{K}$; then $V(K)$ is a $K$-representation. The $G$-space $Y$ is $G$-V -connected if $\pi_{V(K)+m}^{K}(Y)$ is zero for each subgroup $K$ of $G$ and each integer $m$ with $0 \leq m \leq\left|V^{K}\right|$. Similarly, the $G$-space $Y$ is homologically $G-V$-connected if $H_{V(K)+m}^{G}(Y)$ is zero for each subgroup $K$ of $G$ and each integer $m$ with $0 \leq m \leq\left|V^{K}\right|$.

(v) Let $V$ be a $G$-representation. A $G$-0*-equivalence $f: Y \rightarrow Z$ is said to be a $G$ - $V$-equivalence if, for every subgroup $K$ of $G$, the map

$$
f_{*}: \pi_{V(K)+m}^{K}(Y) \rightarrow \pi_{V(K)+m}^{K}(Z)
$$

is an isomorphism for every integer $m$ with $0 \leq m<\left|V^{K}\right|$ and an epimorphism for $m=\left|V^{K}\right|$. A homology $G$ - $V$-equivalence is defined similarly. A $G$-pair $(Y, B)$ is $G-V$-connected (or homologically $G-V$-connected) if the inclusion of $B$ into $Y$ is a $G-V$-equivalence (or a homology $G-V$-equivalence).

(vi) Let $B$ be a $G$-subspace of $Y$ and let $K$ be a subgroup of $G$. If the inclusion of $B$ into $Y$ is a $K-0^{*}$-equivalence but not a weak $K$-equivalence, then the $K$-connectivity $c^{K}(Y, B)$ of the pair $(Y, B)$ is the largest integer $n$ such that, for every subgroup $J$ of $K$,

$$
\left(f^{J}\right)_{*}: \pi_{m}\left(B^{J}\right) \rightarrow \pi_{m}\left(Y^{J}\right)
$$

is an isomorphism for every integer $m$ with $0 \leq m<n$ and an epimorphism for $m=n$. If the inclusion of $B$ in $Y$ is a weak $K$-equivalence, then $c^{K}(Y, B)$ is $\infty$. If the inclusion of $B$ in $Y$ is not a $K$-0*-equivalence, then $c^{K}(Y, B)$ is -1 . The $K$-connectivity $c^{K} Y$ of a based $G$-space $Y$ is $c^{K}(Y, *)$, where * is the basepoint of $Y$. Note that if $F i$ is the homotopy fiber of the inclusion $i: B \rightarrow Y$, then $c^{K}(Y, B)$ is $1+c^{K} F i$. The dimension functions with values $c^{K}(Y, B)$ and $c^{K} Y$ at $K$ are denoted $c^{*}(Y, B)$ and $c^{*} Y$.

The prefix " $G$-" is deleted from all of our notations for connectivity and equivalence whenever the omission should not lead to confusion.

Waner's result, which we extend to compact Lie groups, equates $V$-connectivity and $\left|V^{*}\right|$-connectivity. This equation suggests the possibility of using a multitude of representations to obtain bounds for $c^{*} Y$. Any one representation might provide a good estimate of $c^{K} Y$ for only a few subgroups $K$, but a collection of representations might together provide a complete picture of $c^{*} Y$. 
Lemma 1.2. Let $Y$ and $Z$ be G-spaces, $(Y, B)$ be a G-pair, $f: Y \rightarrow Z$ be a $G$-map, and $V$ be a G-representation. Then the following assertions are true:

(i) The space $Y$ is $V$-connected if and only if it is $\left|V^{*}\right|$-connected.

(ii) The G-pair $(Y, B)$ is $V$-connected if and only if it is $\left|V^{*}\right|$-connected.

(iii) The map $f$ is a $V$-equivalence if and only if it is $a\left|V^{*}\right|$-equivalence.

Hereafter, we make constant implicit use of this lemma by employing the notions of $V$ - and $\left|V^{*}\right|$-connectivity interchangeably.

Our first form of the unstable equivariant Hurewicz Isomorphism Theorem relates the unmodified equivariant homotopy groups of a $G$-space $Y$ to the equivariant homology of $Y$. The proof of this theorem, given in $\S 3$, can easily be extended to give the obvious relative version of the theorem.

Theorem 1.3. Let $Y$ be a simply G-connected based G-CW complex and $V$ be a G-representation with $\left|V^{G}\right| \geq 2$.

(i) If, for all $J<K \leq G$,

(a) $\left|(V-1)^{K}\right| \leq c^{K} Y$ and

(b) $1+\left|V^{K}\right| \leq c^{J} Y$,

then the Hurewicz map $h: \pi_{V}^{G}(Y) \rightarrow H_{V}^{G}(Y)$ is an isomorphism. Moreover, if all the inequalities in (a) are strict, then both groups are zero.

(ii) If, for all $J<K \leq G$,

(a) $\left|(V-2)^{K}\right| \leq c^{K} Y$ and

(b) $\left|V^{K}\right| \leq c^{J} Y$,

then the Hurewicz map $h: \pi_{V}^{G}(Y) \rightarrow H_{V}^{G}(Y)$ is an epimorphism.

Condition (a) in each part of this theorem is exactly the condition that one would expect from the nonequivariant Hurewicz theorem. However, condition (b) in both parts is extremely restrictive. It is necessary because the group $H_{V}^{G}(Y)$ is stable with respect to suspension by every $G$-representation $W$; whereas condition (a) can, at best, only ensure that the group $\pi_{V}^{G}(Y)$ is stable with respect to suspension by the representation $V$. To eliminate condition (b), we must give up the epimorphism half of the theorem. Moreover, for the first half of the theorem, we must accept a comparison of a modification of the equivariant homotopy groups with the equivariant homology groups. In order to describe the required modifications to the homotopy groups, we must first describe the full structure of the equivariant homotopy and homology groups of $Y$. These groups are actually contravariant functors from certain categories whose objects are the orbits $G / K$ for the group $G$.

Definition 1.4. (i) The orbit category $\mathscr{O}_{G}$ has as objects the orbits $G / K$. The set of morphisms in $\mathscr{O}_{G}$ from $G / K$ to $G / J$ is the set of $G$-homotopy classes of $G$-maps from $G / K$ to $G / J$.

(ii) Let $V$ be a finite- or countably infinite-dimensional $G$-representation. The $V$-Burnside category $\mathscr{B}_{G}(V)$ has as its objects the orbits $G / K$. If $V$ is finite dimensional, then the set of morphisms from $G / K$ to $G / J$ is $\left[\Sigma^{V} G / K^{+}, \Sigma^{V} G / J^{+}\right]_{G}$. If $V$ is infinite dimensional, then the set of morphisms is the colimit of the sets $\left[\Sigma^{W} G / K^{+}, \Sigma^{W} G / J^{+}\right]_{G}$, where $W$ runs over the finitedimensional $G$-invariant subspaces of $V$. If $\left|V^{G}\right| \geq 2$, then the morphism sets of $\mathscr{B}_{G}(V)$ are abelian groups. Recall from $[15, \mathrm{p}$. 11] that a $G$-universe 
is a countably infinite-dimensional $G$-representation which contains the trivial $G$-representation and which contains infinitely many copies of each of its finite-dimensional subrepresentations. Also recall that a complete G-universe is just a $G$-universe that contains infinitely many copies of every irreducible $G$-representation. When $V$ is a $G$-universe, the set of morphisms in $\mathscr{B}_{G}(V)$ from $G / K$ to $G / J$ is just the set of $V$-stable $G$-maps from the $V$-suspension spectrum of $G / K^{+}$to that of $G / J^{+}$. We write $\mathscr{B}_{G}$ for $\mathscr{B}_{G}(V)$ when $V$ is a complete $G$-universe.

(iii) Stabilization gives a functor $s: \mathscr{O}_{G} \rightarrow \mathscr{B}_{G}(V)$ for any $G$-representation $V$. If $W \subset V$, then stabilization gives a functor $s: \mathscr{B}_{G}(W) \rightarrow \mathscr{B}_{G}(V)$.

In $\S 3$ it will be shown that this second functor is often an isomorphism of categories. In particular, if $\left|V^{G}\right| \geq 2$, then $s: \mathscr{B}_{G}(V) \rightarrow \mathscr{B}_{G}(V+n)$ is an isomorphism.

The orbit category $\mathscr{O}_{G}$, for finite groups $G$, appears in Bredon's work [1] on equivariant ordinary cohomology theories. Illman introduces $\mathscr{O}_{G}$ for any compact Lie group $G$ in his extension $[9,10]$ of Bredon's work. The Burnside category $\mathscr{B}_{G}$ is an elementary extension of the Burnside ring and is described in $\S \mathrm{V} .9$ of [15].

Definition 1.5. (i) A coefficient system is a contravariant functor from $\mathscr{O}_{G}$ to the category $A b$ of abelian groups. The category of all such functors (together with the natural transformations among these functors) is denoted $\mathscr{C}_{G}$.

(ii) If $V$ is a $G$-representation with $\left|V^{G}\right| \geq 2$, then a $V$-Mackey functor is a contravariant additive functor from the category $\mathscr{B}_{G}(V)$ to $A b$. The category of all such functors is denoted $\mathscr{M}_{G}(V)$. If $V$ is a complete $G$-universe, then such functors are called Mackey functors and the category is denoted $\mathscr{M}_{G}$. When $G$ is finite, this definition of a Mackey functor is equivalent to that normally given in representation theory $[5,11,14,15]$. If $\mathbb{R}^{n}$ is the $n$-dimensional trivial $G$-representation, then $\mathbb{R}^{n}$-Mackey functors are simply coefficient systems because the morphism sets of $\mathscr{B}_{G}\left(\mathbb{R}^{n}\right)$ are the free abelian groups generated by the corresponding morphism sets of $\mathscr{O}_{G}[18,22]$. Thus, coefficient systems are a particular type of $V$-Mackey functors.

(iii) Precomposition by the functors $s$ of Definition 1.4(iii) gives functors

$$
s^{*}: \mathscr{M}_{G}(V) \rightarrow \mathscr{C}_{G}, \quad s^{*}: \mathscr{M}_{G}(V) \rightarrow \mathscr{M}_{G}(W)
$$

for any $G$-representation $V$ and any inclusion $W \subset V$ of $G$-representations. The first functor is the special case of the second in which $W$ is taken to be a copy of $\mathbb{R}^{2}$ in $V$. Whenever $s: \mathscr{B}_{G}(W) \rightarrow \mathscr{B}_{G}(V)$ is an isomorphism, we identify $V$ - and $W$-Mackey functors via the induced isomorphism $s^{*}$.

Examples 1.6. (i) For any $G$-representation $V$ and any based $G$-space $Y$, the homotopy set $\pi_{V}^{G}(Y)$ may be converted into a contravariant functor from the orbit category $\mathscr{O}_{G}$ to the category Sets $_{*}$ of pointed sets by defining its value on the object $G / K$ to be

$$
\left[G / K^{+} \wedge S^{V}, Y\right]_{G} \cong\left[S^{V}, Y\right]_{K}=\pi_{V}^{K}(Y) .
$$

The effect of a morphism $f: G / K \rightarrow G / J$ in $\mathscr{O}_{G}$ is simply that of precomposition by the suspension of $f$ regarded as a map between $G$-spaces. We denote this functor by $\pi_{V}^{G}(Y)$. If $\left|V^{G}\right| \geq 2$, then this functor is a $V$-Mackey functor. 
Unfortunately, if $\left|V^{G}\right|=1, \pi_{V}^{G}(Y)$ is not necessarily a functor from $\mathscr{B}_{G}(V)$ to the category $G r p$ of groups. The value of $\pi_{V}^{G}(Y)$ at any orbit $G / K$ is a group. However, some of the maps in $\mathscr{B}_{G}(V)$ are not suspensions of maps and so need not induce group homomorphisms between the values of $\pi_{V}^{G}(Y)$. Note that $c^{G} Y$ is the largest integer $n$ such that $\pi_{m}^{G}(Y)$ vanishes for all $m \leq n$.

(ii) Similarly, if $V$ is a $G$-representation that contains at least one copy of the trivial representation and $(Y, B)$ is a $G$-pair, then the set $\pi_{V}^{G}(Y, B)$ can be converted into a contravariant functor $\pi_{V}^{G}(Y, B)$ into Sets $s_{*}$. Unfortunately, since $\pi_{V}^{G}(Y, B)$ is defined in terms of $\pi_{V-1}^{G}(F i)$, its natural domain category is $\mathscr{B}_{G}(V-1)$ instead of $\mathscr{B}_{G}(V)$. This would be a real inconvenience if these two categories were not isomorphic when $\left|V^{G}\right| \geq 3$. Notice that, if $\left|V^{G}\right|=2$ and $\pi_{V-1}^{G}(B)$ is zero, then $\pi_{V}^{G}(Y, B)$ is a $V$-Mackey functor because it is the cokernel of the natural transformation $\pi_{V}^{G}(B) \rightarrow \pi_{V}^{G}(Y)$ between $V$-Mackey functors.

(iii) The equivariant ordinary homology groups with Burnside ring coefficients of a $G$-space $Y$ or a $G$-pair $(Y, B)$ may be converted into Mackey functors by assigning to the orbit $G / K$ the homology of the space $Y$ (or the pair $(Y, B)$ ) with respect to the subgroup $K$. We denote these functors by $\mathbf{H}_{*}^{G}(Y)$ and $\mathbf{H}_{*}^{G}(Y, B)$.

(iv) Recall that Mackey functors are $U$-Mackey functors for a complete $G$ universe $U$. Such a universe contains any $G$-representation $V$. Thus, choosing a copy of $V$ in $U$ gives a functor $s^{*}$ from the category of Mackey functors to the category of $V$-Mackey functors. Via $s^{*}, \mathbf{H}_{V}^{G}(Y)$ and $\mathbf{H}_{V}^{G}(Y, B)$ may be regarded as $V$-Mackey functors. Then the absolute and relative Hurewicz maps are the natural transformations

$$
h: \boldsymbol{\pi}_{V}^{G}(Y) \rightarrow s^{*} \mathbf{H}_{V}^{G}(Y) \quad \text { and } \quad h: \boldsymbol{\pi}_{V}^{G}(Y, B) \rightarrow s^{*} \mathbf{H}_{V}^{G}(Y, B) .
$$

The functor $s^{*}$ has a left adjoint $s_{*}$ which converts $V$-Mackey functors into Mackey functors (see Lemma 4.5). There are adjoint Hurewicz maps

$$
\tilde{h}: s_{*} \boldsymbol{\pi}_{V}^{G}(Y) \rightarrow \mathbf{H}_{V}^{G}(Y) \text { and } \tilde{h}: s_{*} \boldsymbol{\pi}_{V}^{G}(Y, B) \rightarrow \mathbf{H}_{V}^{G}(Y, B) .
$$

The second form of the unstable Hurewicz Isomorphism Theorem gives conditions under which these adjoint Hurewicz maps are isomorphisms.

Theorem 1.7. Let $Y$ be a based $G-C W$ complex and let $V$ be a G-representation such that $\left|V^{G}\right| \geq 2$. Then the following two conditions are equivalent:

(a) $Y$ is $(V-1)$-connected.

(b) $Y$ is simply G-connected and homologically $(V-1)$-connected.

Moreover, either of these conditions implies that the map

$$
\tilde{h}: s_{*} \boldsymbol{\pi}_{W}^{G}(Y) \rightarrow \mathbf{H}_{W}^{G}(Y)
$$

is an isomorphism for any G-representation $W$ with $2^{*} \leq\left|W^{*}\right| \leq\left|V^{*}\right|$. If $W$ is any G-representation with $\left|W^{*}\right|<\left|V^{*}\right|$, then either of the conditions above implies that both $\boldsymbol{\pi}_{W}^{G}(Y)$ and $\mathbf{H}_{W}^{G}(Y)$ are zero.

Theorem 1.8. Let $(Y, B)$ be a based $G-C W$ pair with both $Y$ and $B$ simply $G$-connected and let $V$ be a G-representation such that $\left|V^{G}\right| \geq 2$. Then the following two conditions are equivalent:

(a) $(Y, B)$ is $(V-1)$-connected. 
(b) $(Y, B)$ is homologically $(V-1)$-connected.

Moreover, either of these conditions implies that, for any G-representation $W$ with $2^{*} \leq\left|W^{*}\right| \leq\left|V^{*}\right|, \pi_{W}^{G}(Y, B)$ is a W-Mackey functor (instead of just a $(W-1)$-Mackey functor) and the map

$$
\tilde{h}: s_{*} \boldsymbol{\pi}_{W}^{G}(Y, B) \rightarrow \mathbf{H}_{W}^{G}(Y, B)
$$

is an isomorphism, where $s_{*}$ is the functor associated to an inclusion of $W$ into a complete G-universe. If $\left|W^{*}\right|<\left|V^{*}\right|$ and $(Y, B)$ is $(V-1)$-connected, then both $\boldsymbol{\pi}_{W}^{G}(Y, B)$ and $\mathbf{H}_{W}^{G}(Y, B)$ are zero.

The nonequivariant Hurewicz theorem is often used to argue that, because the homology groups of a space vanish in a certain range, the homotopy groups must also vanish in that range. The presence of $s_{*} \pi_{W}^{G}(Y)$ instead of $\pi_{W}^{G}(Y)$ in Theorem 1.7 might seem to preclude similar arguments in the equivariant context, since the obvious conclusion would be only that $s_{*} \pi_{W}^{G}(Y)$ vanishes when $\mathbf{H}_{W}^{G}(Y)$ does. The following result, equating the vanishing of $s_{*} \boldsymbol{\pi}_{W}^{G}(Y)$ and $\pi_{W}^{G}(Y)$, eliminates this difficulty.

Proposition 1.9. Let $W \subset V$ be G-representations with $\left|W^{G}\right| \geq 2, N$ be a $W$-Mackey functor, and $s_{*}: \mathscr{M}_{G}(W) \rightarrow \mathscr{M}_{G}(V)$ be the functor associated to the inclusion of $W$ in $V$. If $K$ is a subgroup of $G$ such that $N(G / J)=0$ for every proper subgroup $J$ of $K$, then

$$
\left(s_{*} N\right)(G / K)=N(G / K) .
$$

In particular, $\left(s_{*} N\right)(G /\{e\})=N(G /\{e\})$. Also, if $s_{*} N=0$, then $N=0$.

The following homological criterion for contractibility is an obvious consequence of our absolute Hurewicz theorem.

Proposition 1.10. Let $Y$ be a simply G-connected $G-C W$ complex. If there is a $G$-representation $V$ such that $H_{V+m}^{K}(Y)=0$ for every subgroup $K$ of $G$ and every integer $m$, then $Y$ is $G$-contractible.

There is, of course, an equivariant Whitehead theorem corresponding to our equivariant Hurewicz theorems.

Theorem 1.11. Let $Y$ and $Z$ be G-connected G-CW complexes, $f: Y \rightarrow Z$ be a G-map, and $V$ and $W$ be G-representations with $\left|W^{*}\right| \leq\left|V^{*}\right|$. If $f$ is a $V$-equivalence, then $f$ is also a $W$-equivalence and a homology $W$ equivalence. Moreover, if $Y$ and $Z$ are simply G-connected and $f$ is a homology $V$-equivalence, then $f$ is a $V$-equivalence.

The two real disappointments in our results are the lack of any analog of Whitehead's epimorphism result [23] in Theorems 1.7 and 1.8 and the lack of any results for representations $V$ with $\left|V^{G}\right| \leq 1$. We conclude this section with a counterexample and some remarks related to these disappointments.

Example 1.12. Let $p$ be a prime and $n \geq 3$. Let $G=\mathbb{Z} / p, V$ be a nontrivial irreducible complex representation of $G$, and $Y$ be $\Sigma^{n} S V^{+}$. An equivariant analog of Whitehead's result would require that the map

$$
\tilde{h}: s_{*} \boldsymbol{\pi}_{n+1}^{G}(Y) \rightarrow \mathbf{H}_{n+1}^{G}(Y)
$$


be an epimorphism. Since $G$ acts freely off the basepoint of $Y$, it is easy to check that, for $\varepsilon=0$ or 1 , the value of the coefficient system $\pi_{n+\varepsilon}^{G}(Y)$ at the orbit $G / K$ is given by

$$
\boldsymbol{\pi}_{n+\varepsilon}^{G}(Y)(G / K)= \begin{cases}0, & \text { if } K=G, \\ \mathbb{Z}, & \text { if } K=\{e\} \text { and } \varepsilon=0, \\ \mathbb{Z} \oplus \mathbb{Z} / 2, & \text { if } K=\{e\} \text { and } \varepsilon=1\end{cases}
$$

For $\varepsilon=0$ or 1 , the Mackey functor $\mathbf{H}_{n+\varepsilon}^{G}(Y)$ can be computed using the cofiber sequence

$$
G^{+} \rightarrow S V^{+} \rightarrow \Sigma G^{+} \rightarrow \Sigma G^{+},
$$

in which the first map is the inclusion of an orbit and the third map is the difference of the identity map and the $G$-map sending $e \in G$ to some generator $\tau \in G[12,13]$. For either value of $\varepsilon$, the value of $\mathbf{H}_{n+\varepsilon}^{G}(Y)$ at both the orbit $G / e$ and the orbit $G / G$ is $\mathbb{Z}$. If $\varepsilon=0$, then the transfer map from $\mathbf{H}_{n+\varepsilon}^{G}(Y)(G / e)$ to $\mathbf{H}_{n+\varepsilon}^{G}(Y)(G / G)$ is the identity and the restriction map from $\mathbf{H}_{n+\varepsilon}^{G}(Y)(G / G)$ to $\mathbf{H}_{n+\varepsilon}^{G}(Y)(G / e)$ is multiplication by $p$. On the other hand, if $\varepsilon=1$, the transfer map is multiplication by $p$ and the restriction map is the identity. Note that $\pi_{n+1}^{G}(Y)$ may be regarded as the direct sum of the coefficient system $\pi_{n}^{G}(Y)$ and a 2-torsion coefficient system. Since the Hurewicz theorem says that $s_{*} \boldsymbol{\pi}_{n}^{G}(Y)$ is isomorphic to the Mackey functor $\mathbf{H}_{n}^{G}(Y), s_{*} \boldsymbol{\pi}_{n+1}^{G}(Y)$ must be isomorphic to the direct sum of the Mackey functor $\mathbf{H}_{n}^{G}(Y)$ and a 2-torsion Mackey functor. It follows by an easy computation that there is no epimorphic natural transformation from $s_{*} \pi_{n+1}^{G}(Y)$ to $\mathbf{H}_{n+1}^{G}(Y)$. Thus, there can be no analog of Whitehead's result for Theorem 1.7.

Remarks 1.13. The nonequivariant analog of the Hurewicz Isomorphism Theorem for a representation $V$ with $\left|V^{G}\right|=0$ is, of course, the assertion that $H_{0}(X)$ is the free abelian group generated by the set of path components of $X$. The analog for $\left|V^{G}\right|=1$ is the assertion that, for a path connected space $X$, the map

$$
h: \pi_{1}(X) \rightarrow H_{1}(X)
$$

is just the projection of a group onto its abelianization. The proofs of these two results are rather different from the proof of the Hurewicz theorem for higher dimensions; they depend very heavily on the geometric simplicity of 0 - and 1 -simplices. A $G$-representation $V$ with $\left|V^{G}\right| \leq 1$ certainly need not share in this simplicity. Thus, it seems that the proof of an unstable Hurewicz theorem applicable to a general representation $V$ of this form may be quite difficult. On the other hand, we may regard $\pi_{0}^{G}(Y)$ and $\pi_{1}^{G}(Y)$ as functors from $\mathscr{O}_{G}$ to the categories Sets $s_{*}$ of pointed sets and Grp of groups, respectively. There are then suspension natural transformations

$$
\sigma_{0}: \pi_{0}^{G}(Y) \rightarrow r_{0}^{*} \pi_{2}^{G}\left(\Sigma^{2} Y\right), \quad \sigma_{1}: \pi_{1}^{G}(Y) \rightarrow r_{1}^{*} \pi_{2}^{G}(\Sigma Y),
$$

where $r_{0}^{*}$ and $r_{1}^{*}$ are the obvious forgetful functors. The behavior of these two natural transformation, which describe the effect of suspension on the homotopy sets of the various fixed point subspaces of $Y$, is quite obvious. At each orbit $G / H, \sigma_{0}$ simply injects a pointed set into the free abelian group it generates (the basepoint of the pointed set is sent to the zero of the group). Similarly, if $Y$ is $G$-connected, then, at each orbit, $\sigma_{1}$ is just the projection of a group onto 
its abelianization. Theorem 1.7 applies to $\pi_{2}^{G}\left(\Sigma^{2} Y\right)$ and, if $Y$ is $G$-connected, to $\pi_{2}^{G}(\Sigma Y)$. From this, we obtain Hurewicz isomorphisms

$$
h: s_{*} r_{*}^{0} \boldsymbol{\pi}_{0}^{G}(Y) \rightarrow \mathbf{H}_{0}^{G}(Y)
$$

and, if $Y$ is $G$-connected,

$$
h: s_{*} r_{*}^{1} \boldsymbol{\pi}_{1}^{G}(Y) \rightarrow \mathbf{H}_{1}^{G}(Y) .
$$

Here, $s_{*}$ is the functor arising from an inclusion of $\mathbb{R}^{2}$ into a complete $G$ universe, and $r_{*}^{0}$ and $r_{*}^{1}$ are the left adjoints of $r_{0}^{*}$ and $r_{1}^{*}$.

\section{THE STABLE HUREWICZ ISOMORPHISM}

Our unstable equivariant Hurewicz isomorphism theorems are derived from a stable equivariant Hurewicz isomorphism theorem and an equivariant Freudenthal Suspension Theorem. Recall that $\operatorname{RO}(G)$-graded equivariant ordinary cohomology with Mackey functor coefficients was defined in [14] by constructing a representing Eilenberg-Mac Lane $G$-spectrum. This spectrum must be indexed on a complete $G$-universe $U$. Thus, throughout this section, we assume that all spectra are indexed on this complete $G$-universe. Roughly speaking, this is just the assumption that our $G$-spectra are indexed on all $G$-representations. If Burnside ring coefficients are used in our ordinary theory, then the representing $G$-spectrum $H A$ is a commutative ring spectrum, whose unit $S^{0} \rightarrow H A$ induces the stable Hurewicz map

$$
h: \pi_{*}^{G}(D) \rightarrow H_{*}^{G}(D) .
$$

The equivariant stable Hurewicz isomorphism theorem specifies the dimensions in which this map is an isomorphism between the equivariant stable homotopy and homology of a $(-1)$-connected $G$-spectrum.

Theorem 2.1. Let $D$ be a $G$-CW spectrum such that $\pi_{n}^{K}(D)=0$ for all subgroups $K$ of $G$ and all $n<0$. Let $\alpha$ be an element of $\mathrm{RO}(G)$. Then the following assertions are true:

(i) If $\left|\alpha^{K}\right|<0$ for all subgroups $K$ of $G$, then $\pi_{\alpha}^{G}(D)$ and $H_{\alpha}^{G}(D)$ are both zero.

(ii) If $\left|\alpha^{K}\right| \leq 0$ for all subgroups $K$ of $G$, then the stable Hurewicz map $h: \pi_{\alpha}^{G}(D) \rightarrow H_{\alpha}^{G}(D)$ is an isomorphism.

(iii) If $\left|\alpha^{K}\right| \leq 1$ for all subgroups $K$ of $G$, then the stable Hurewicz map $h$ is an epimorphism.

Remarks 2.2. The epimorphism portion of this theorem has a curious implication for the equivariant stable homotopy groups of spheres. Let $G$ be a finite group, $\eta$ be a nontrivial complex one-dimensional representation of $G$, and $\beta$ be a virtual representation of $G$ such that $\left|\beta^{K}\right|=0$ for every subgroup $K$ of $G$. The Hurewicz map

$$
\pi_{\beta+\eta-1}^{G}\left(\Sigma^{\infty} S^{0}\right) \rightarrow H_{\beta+\eta-1}^{G}\left(S^{0}\right)
$$

is onto by Theorem 2.1(iii). Thus, any nonvanishing result for $H_{\beta+\eta-1}^{G}\left(S^{0}\right)$ implies the existence of nonzero elements in $\pi_{\beta+\eta-1}^{G}\left(\Sigma^{\infty} S^{0}\right)$. The methods used in [13] to compute $\mathbf{H}_{G}^{*}\left(S^{0}\right)$ for $G=\mathbb{Z} / p$ can be applied to the computation of 
$H_{\beta+\eta-1}^{G}\left(S^{0}\right)$ for any finite group $G$. Let $J$ be the kernel of $\eta$. Then there is an exact sequence

$$
\mathbf{H}_{\beta}^{G}\left(S^{0}\right) \stackrel{\gamma}{\longrightarrow} h_{G / J} \mathbf{H}_{\beta}^{G}\left(S^{0}\right) \rightarrow \mathbf{H}_{\beta+\eta-1}^{G}\left(S^{0}\right) \rightarrow 0,
$$

where $h_{G / J} \mathbf{H}_{\beta}^{G}\left(S^{0}\right)$, Dress's zero-dimensional cohomology of $\mathbf{H}_{\beta}^{G}\left(S^{0}\right)$ with respect to $G / J[5,11]$, is the equalizer of the pair of transfers

$$
\mathbf{H}_{\beta}^{G}\left(G / J^{+}\right) \underset{\tau_{2}}{\stackrel{\tau_{1}}{\rightrightarrows}} \mathbf{H}_{\beta}^{G}\left(G / J^{+} \wedge G / J^{+}\right) \text {. }
$$

The map $\gamma$ is the unique map arising from the fact that the transfer

$$
\tau: \mathbf{H}_{\beta}^{G}\left(S^{0}\right) \rightarrow \mathbf{H}_{\beta}^{G}\left(G / J^{+}\right)
$$

equalizes $\tau_{1}$ and $\tau_{2}$. Since $\left|\beta^{K}\right|=0$ for all $K \leq G, \mathbf{H}_{\beta}^{G}\left(S^{0}\right)$ and $\boldsymbol{\pi}_{\beta}^{G}\left(\Sigma^{\infty} S^{0}\right)$ are isomorphic by Theorem 2.1(ii). The description of $\pi_{\beta}^{0}\left(\Sigma^{\infty} S^{0}\right)$ given in [4] may be used to compute $\mathbf{H}_{\beta+\eta-1}^{G}\left(S^{0}\right)$. If $J$ is not the trivial subgroup $\{e\}$, then $\mathbf{H}_{\beta+\eta-1}^{G}\left(S^{0}\right)$ is nonzero under fairly general conditions. In particular, if $G$ is abelian, then $H_{\beta+\eta-1}^{G}\left(S^{0}\right)$ is the cokernel of the map

$$
\tau: H_{\beta}^{G}\left(S^{0}\right) \rightarrow H_{\beta}^{G}\left(G / J^{+}\right) .
$$

For example, if $G=\mathbb{Z} / p^{2}$ and $J=\mathbb{Z} / p$, then $H_{\beta+\eta-1}^{G}\left(S^{0}\right)$ is $\mathbb{Z} / p$. Thus, there are nonzero elements in the equivariant stable homotopy groups of spheres in this generalized dimension one. More surprising, these elements are detected by homology. The epimorphism result (Theorem 1.3(ii)) in our first unstable Hurewicz theorem gives some indication of when these elements in the equivariant stable stems actually appear in the unstable equivariant homotopy groups of spheres.

In Theorem 2.6 of [13], it was shown that, if $G=\mathbb{Z} / p$ and $Y$ is a generalized $G$-CW complex constructed from the unit disks of complex $G$-representations satisfying certain dimensional restrictions, then the equivariant ordinary cohomology of $Y$ is free as an $\mathrm{RO}(G)$-graded algebra over $\mathbf{H}_{*}^{G}\left(S^{0}\right)$. The proof turns on the failure of equivariant ordinary cohomology to detect the attaching maps of the cells of $Y$. The fact that, for $G=\mathbb{Z} / p^{2}$, the Hurewicz map is nonzero in dimensions of the form $\beta+\eta-1$ indicates that this freeness result from [13] cannot be generalized beyond the groups $\mathbb{Z} / p$. Preliminary calculations even suggest that the attaching maps of the cells of certain complex projective spaces with linear $\mathbb{Z} / p^{2}$ actions are detected by homology groups in dimensions of the form $\beta+\eta-1$. Thus, Proposition 3.1 of [13], which asserts the freeness of the cohomology of complex projective spaces with linear $\mathbb{Z} / p$ actions, may not generalize to groups other than $\mathbb{Z} / p$.

The remainder of this section is devoted to the proof of Theorem 2.1. The two technical results below establish the behavior of the Hurewicz map in important special cases. If $G$ is finite, then the first result is a trivial consequence of the dimension axiom and the generalization of Wirthmüller's isomorphism given in Corollary II.6.5 of [15]. More effort is required when $G$ is not finite.

Lemma 2.3. Let $K$ and $L$ be subgroups of $G$ and let $n$ be a positive integer. Then the following assertions are true:

(i) $\left[\Sigma^{\infty} G / K^{+}, \Sigma^{n} \Sigma^{\infty} G / L^{+}\right]_{G}=0$. 
(ii) $\left[\Sigma^{\infty} G / K^{+}, \Sigma^{n} \Sigma^{\infty} G / L^{+} \wedge H A\right]_{G}=0$.

(iii) The map

$$
\left[\Sigma^{\infty} G / K^{+}, \Sigma^{\infty} G / L^{+}\right]_{G} \rightarrow\left[\Sigma^{\infty} G / K^{+}, \Sigma^{\infty} G / L^{+} \wedge H A\right]_{G},
$$

induced by the unit $e: S \rightarrow H A$, is an isomorphism.

Proof. Part (i) is a special case of Proposition 3.2. For the rest, recall that the spectrum $H A$ was obtained in [14] as the spectrum representing zerodimensional equivariant ordinary cohomology with Burnside ring coefficients. The dimension axiom implies that $H A$ is $G-(-1)$-connected. Thus, $H A$ may be assumed to be a $G$-CW spectrum whose bottom cells are in dimension zero by Proposition I.7.14 of [15]. Since $H_{G}^{0}\left(S^{0}\right)$ is the Burnside ring, which is the stable zero stem, the zero skeleton of $H A$ may be taken to be $S^{0}$. With this choice, no 1-cells are required to form $H A$ and the unit $S^{0} \rightarrow H A$ is the inclusion of the 1-skeleton into $H A$. Parts (ii) and (iii) follow from this observation and part (i) by an induction argument over the skeleton of $H A$.

Proposition 2.4. Let $X$ and $Y$ be based $G-C W$ complexes such that $X$ is finite dimensional. Let $\varepsilon$ be 0,1 , or 2 and assume that, for each subgroup $K$ of $G$, the dimension of each $G / K$-cell of $X$ is less than or equal to $c^{K} Y+\varepsilon$. Then the following assertions are true:

(i) If $\varepsilon=0$, then $\left[\Sigma^{\infty} X, \Sigma^{\infty} Y\right]_{G}$ and $\left[\Sigma^{\infty} X, \Sigma^{\infty} Y \wedge H A\right]_{G}$ are both zero.

(ii) If $\varepsilon=1$, then the map

$$
h:\left[\Sigma^{\infty} X, \Sigma^{\infty} Y\right]_{G} \rightarrow\left[\Sigma^{\infty} X, \Sigma^{\infty} Y \wedge H A\right]_{G},
$$

induced by the unit of $H A$, is an isomorphism.

(iii) If $\varepsilon=2$, then the map $h$ is an epimorphism.

Proof. Part (i) follows from an induction argument on the skeleton of $X$. For part (ii), observe that $h$ induces a map of the long exact sequences obtained by applying the functors $\left[?, \Sigma^{\infty} Y\right]_{G}$ and $\left[?, \Sigma^{\infty} Y \wedge H A\right]_{G}$ to the cofiber sequence

$$
X^{n} \rightarrow X^{n+1} \rightarrow X^{n+1} / X^{n},
$$

where $X^{n}$ is the $n$-skeleton of $X$. An induction argument on the skeleton of $X$ reduces the proof to the case $X=G / K^{+} \wedge S^{n}$, with $n \leq c^{K} Y+1$. The isomorphism

$$
\left[\Sigma^{\infty} G / K^{+} \wedge S^{n}, \Sigma^{\infty} Y \wedge H A\right]_{G} \cong\left[\Sigma^{\infty} S^{n}, \Sigma^{\infty} Y \wedge H A\right]_{K}
$$

and the corresponding isomorphism with $H A$ replaced by $S$ further reduce the proof to the case $K=G$. We may assume that the space $Y$ is a $G-\mathrm{CW}$ complex whose cells are all of dimension greater than or equal to $c^{G} Y+1$. Arguing inductively over the skeleton of $Y$, we obtain part (ii) from Lemma 2.3. For part (iii), we proceed exactly as in part (ii) to reduce the proof to the case $X=S^{n}$ and $Y=\Sigma^{n-1} G / J^{+}$. In the event that $J=G$ or $G=\{e\}, h$ is trivially an epimorphism because, by the cohomology dimension axiom, its range vanishes. Otherwise, Wirthmüller's isomorphism [15] identifies

$$
h:\left[\Sigma^{\infty} S^{n}, \Sigma^{\infty} \Sigma^{n-1} G / J^{+}\right]_{G} \rightarrow\left[\Sigma^{\infty} S^{n}, \Sigma^{\infty} \Sigma^{n-1} G / J^{+} \wedge H A\right]_{G}
$$

with the map

$$
h:\left[\Sigma^{\infty} S^{n}, \Sigma^{\infty} S^{T+n-1}\right]_{J} \rightarrow\left[\Sigma^{\infty} S^{n}, \Sigma^{\infty} S^{T+n-1} \wedge H A\right]_{J},
$$


where $T$ is the $J$-representation given by the tangent space of $G / J$ at the identity coset $e J$. This reduces the proof of part (iii) to an instance of part (iii) for a proper subgroup of $G$. Since we have observed that part (iii) holds for $G=\{e\}$, an inductive argument using the descending chain condition for subgroups completes the proof.

The equivariant stable Hurewicz isomorphism theorem follows easily from this proposition.

Proof of Theorem 2.1. Since $D$ is $G-(-1)$-connected, we may assume that it is a $G$-CW spectrum whose lowest-dimensional cells are in dimension zero (see Proposition I.7.14 of [15]). Arguing inductively over the skeleton of $D$, we can reduce to the case $D=\Sigma^{\infty} G / K^{+} \wedge S^{n}$, where $n \geq 0$. If $\alpha$ is represented by the formal difference $V-W$ of $G$-representations $V$ and $W$, then this case follows from the proposition above with $X=S^{V}$ and $Y=G / K^{+} \wedge S^{W+n}$.

\section{THE STABILIZATION OF HOMOTOPY GROUPS}

Here we discuss the passage from unstable to stable equivariant homotopy groups. Out of this discussion, we obtain the promised generalization of Lemma 2.3(i), two observations about the functors $s$ of Definition 1.4(iii), the proof of the first version of our unstable Hurewicz theorem (Theorem 1.3), and the intuition behind the proof of the second form of our unstable Hurewicz theorem.

If $X$ and $Y$ are based $G$-spaces and $W$ is a $G$-representation, then suspension by $W$ induces a map

$$
\sigma:[X, Y]_{G} \rightarrow\left[\Sigma^{W} X, \Sigma^{W} Y\right]_{G} .
$$

Moreover, for any $G$-universe $U$, there is an "infinite" suspension, or stabilization, map

$$
\sigma:[X, Y]_{G} \rightarrow\left[\Sigma^{\infty} X, \Sigma^{\infty} Y\right]_{G} .
$$

The following definition of the unstable Hurewicz maps explains a part of our interest in these suspension maps.

Definition 3.1. (i) Let $Y$ be a based $G$-space and $V$ be a $G$-representation. The absolute unstable Hurewicz map is the composite

$$
h: \pi_{V}^{G}(Y)=\left[S^{V}, Y\right]_{G} \stackrel{\sigma}{\longrightarrow}\left[\Sigma^{\infty} S^{V}, \Sigma^{\infty} Y\right]_{G}=\pi_{V}^{G}\left(\Sigma^{\infty} Y\right) \stackrel{h}{\longrightarrow} H_{V}^{G}(Y) .
$$

Here, $\pi_{V}^{G}\left(\Sigma^{\infty} Y\right)$ is to be computed with respect to a complete $G$-universe $U$ and the last map is just the stable Hurewicz map induced by the unit $e: S^{0} \rightarrow H A$ of the Eilenberg-Mac Lane spectrum $H A$. Due to its naturality, the unstable Hurewicz map may be regarded as a natural transformation

$$
h: \boldsymbol{\pi}_{V}^{G}(Y) \rightarrow s^{*} \mathbf{H}_{V}^{G}(Y),
$$

where $s^{*}$ is the functor induced by an inclusion of $V$ into $U$.

(ii) Let $(Y, B)$ be a $G$-pair and $V$ be a $G$-representation with $\left|V^{G}\right| \geq 1$. Let $F i$ and $C i$ be the homotopy fiber and cofiber of the inclusion $i: B \rightarrow Y$. The relative unstable Hurewicz map is the composite

$$
\begin{aligned}
h: \pi_{V}^{G}(Y, V) & =\pi_{V-1}^{G}(F i) \stackrel{h}{\rightarrow} H_{V-1}^{G}(F i) \\
& \cong H_{V}^{G}(\Sigma F i) \rightarrow H_{V}^{G}(C i)=H_{V}^{G}(Y, B) .
\end{aligned}
$$


Here, the first map is the absolute unstable Hurewicz map and the last map is induced by the canonical comparison map between $\Sigma F i$ and $C i$. The relative Hurewicz map may, of course, be regarded as the natural transformation between the $(V-1)$-Mackey functors $\boldsymbol{\pi}_{V}^{G}(Y, B)$ and $s^{*} \mathbf{H}_{V}^{G}(Y, B)$, where the functor $s^{*}$ comes from an inclusion of $V-1$ into $U$.

Obviously, to prove our unstable Hurewicz theorems, we must study the stabilization map $\sigma$. Recall from $[15$, p. 11] that an indexing sequence for a $G$-universe $U$ is a sequence $\left\{A_{m}\right\}_{m \geq 0}$ of $G$-subspaces of $U$ with $A_{0}=0$, $A_{m} \subset A_{m+1}$, and $U$ the union of the $A_{m}$. If $X$ and $Y$ are based $G-\mathrm{CW}$ complexes and $X$ is finite, then

$$
\left[\Sigma^{\infty} X, \Sigma^{\infty} Y\right]_{G}=\operatorname{colim}\left[\Sigma^{A_{m}} X, \Sigma^{A_{m}} Y\right]_{G}
$$

where the colimit is taken over the maps induced by suspension by the orthogonal complement of $A_{m}$ in $A_{m+1}$ (see Lemma I.4.8 of [15]). The stabilization map $\sigma$ is the inclusion of the first term in the colimit system. To show that the map $\sigma$ is an isomorphism or an epimorphism, it suffices to show that the stabilization map

$$
\left[\Sigma^{A_{m}} X, \Sigma^{A_{m}} Y\right]_{G} \rightarrow\left[\Sigma^{A_{m+1}} X, \Sigma^{A_{m+1}} Y\right]_{G}
$$

is an isomorphism or epimorphism for $m \geq 0$. Also, to show that the group $\left[\Sigma^{\infty} X, \Sigma^{\infty} Y\right]_{G}$ vanishes, it suffices to show that the groups $\left[\Sigma^{A_{m}} X, \Sigma^{A_{m}} Y\right]_{G}$ vanish for all large $m$.

This observation about $\left[\Sigma^{\infty} X, \Sigma^{\infty} Y\right]_{G}$ suffices for the proof of the generalization of Lemma 2.3(i) promised in $\S 2$.

Proposition 3.2. Let $K$ and $L$ be subgroups of $G$, and let $U$ be any $G$-universe. If $n>0$, then the group $\left[\Sigma^{\infty} G / K^{+}, \Sigma^{n} \Sigma^{\infty} G / L^{+}\right]_{G}$ is zero.

Proof. This group is the colimit of the sets

$$
\left[\Sigma^{A_{m}} G / K^{+}, \Sigma^{n} \Sigma^{A_{m}} G / L^{+}\right]_{G} \cong\left[S^{A_{m}}, \Sigma^{n} \Sigma^{A_{m}} G / L^{+}\right]_{K} .
$$

The $K$-space $S^{A_{m}}$ has a $K$-CW structure in which, for each subgroup $J$ of $K$, the $K / J$ cells of $S^{A_{m}}$ all have dimension no more than $c^{J}\left(\Sigma^{n} \Sigma^{A_{m}} G / L^{+}\right)$. It follows by an induction on the $K$-skeleton of $S^{A_{m}}$ that $\left[S^{A_{m}}, \Sigma^{n} \Sigma^{A_{m}} G / L^{+}\right]_{K}$ is trivial.

The key to the more difficult process of describing when the stabilization map $\sigma$ is an isomorphism or an epimorphism is an equivariant version of the Freudenthal Suspension Theorem. This theorem also provides insight into the behavior of the functors $s$ of Definition 1.4(iii). We employ Namboodiri's version [19] of the equivariant suspension theorem, which we restate here in our notation. Recall that, for a $G$-space $X$, the set $\operatorname{Iso}(X)$ is the set of subgroups of $G$ which are isotropy subgroups of points in $X$.

Theorem 3.3 (Namboodiri). Let $W$ be a G-representation and $Y$ be a based $G$-CW complex such that $c^{K} Y \geq 1$ whenever $\left|W^{K}\right|>0$. Then the suspension map

$$
\sigma:[X, Y]_{G} \rightarrow\left[\Sigma^{W} X, \Sigma^{W} Y\right]_{G}
$$

is an isomorphism if, for all $J<K \leq G$,

(a) $\operatorname{dim} X^{K} \leq 2 c^{K} Y$ when $K \in \operatorname{Iso}(X)$ and

(b) $1+\operatorname{dim} X^{K} \leq c^{J} Y$ when $K \in \operatorname{Iso}(X)$ and $\left|W^{J}\right|>\left|W^{K}\right|$. 
Moreover, $\sigma$ is an epimorphism if, for all $J<K \leq G$,

(a) $\operatorname{dim} X^{K} \leq 1+2 c^{K} Y$ when $K \in \operatorname{Iso}(X)$ and

(b) $\operatorname{dim} X^{K} \leq c^{J} Y$ when $K \in \operatorname{Iso}(X)$ and $\left|W^{J}\right|>\left|W^{K}\right|$.

Condition (b) in both of these assertions corrects an obvious misprint in Theorem 2.3 of [19]. These conditions (b) are vacuously satisfied if the representation $W$ is a trivial $G$-representation. This observation suffices to establish the claim in Definition 1.4(iii) that the functor $s: \mathscr{B}_{G}(V) \rightarrow \mathscr{B}_{G}(V+n)$ is usually an isomorphism.

Lemma 3.4. If $V$ is a G-representation with $\left|V^{G}\right| \geq 2$, then the functor

$$
s: \mathscr{B}_{G}(V) \rightarrow \mathscr{B}_{G}(V+n)
$$

is an isomorphism of categories.

When $W$ is not a trivial representation, the (b) conditions in Namboodiri's theorem are rather strong. The need to satisfy them accounts for the very stringent and unsatisfactory conditions (b) in our first unstable Hurewicz theorem (Theorem 1.3).

Proof of Theorem 1.3. Note that the hypotheses of this theorem are stable in the sense that, if they are satisfied by $V$ and $Y$, then they are satisfied by $V+W$ and $\Sigma^{W} Y$ for any $G$-representation $W$. The isotropy subgroup restrictions present in conditions (a) and (b) of Namboodiri's theorem have been omitted in the hypotheses of Theorem 1.3 to ensure this stability. Thus the conditions in part (i) of Theorem 1.3 imply that the stabilization map

$$
\sigma: \pi_{V}^{G}(Y) \rightarrow \pi_{V}^{G}\left(\Sigma^{\infty} Y\right)
$$

is an isomorphism. Here the stable group is computed with respect to a complete $G$-universe. Similarly, the conditions in part (ii) imply that this stabilization map $\sigma$ is an epimorphism. In both parts of Theorem 1.3, condition (a) ensures that the hypotheses of the appropriate part of Proposition 2.4 are satisfied so that the stable Hurewicz map

$$
h: \pi_{V}^{G}\left(\Sigma^{\infty} Y\right) \rightarrow H_{V}^{G}(Y)
$$

is an isomorphism or epimorphism. The unstable Hurewicz map is the composite of $\sigma$ and the stable Hurewicz map $h$.

In the proof of an unstable Hurewicz theorem, the suspension theorem is applied to the group

$$
\pi_{V}^{G}(Y)=\left[S^{V}, Y\right]_{G}
$$

under the assumption that, for every subgroup $K$ of $G,\left|V^{K}\right| \leq 1+c^{K} Y$. The key to our proof of the second type of the unstable Hurewicz theorem is the observation that, in this context, it is much easier to satisfy condition (b) of the suspension theorem if $W$ is a subrepresentation of $n V$, for some $n \geq 1$. Thus, the proof of the second form of the isomorphism theorem is in two steps. In the first step, which is carried out in this section and in $\S 5$, we study the stabilization of $\pi_{V}^{G}(Y)$ with respect to $V$. In the second step, which is carried out in $\S 6$, we study the stabilization with respect to all the irreducible representations of $G$ missing from $V$. 
To understand why it is easier to deal with the suspension of $\pi_{V}^{G}(Y)$ by a subrepresentation $W$ of $n V$, notice that, if $J<K \leq G$ and $\left|W^{J}\right| \geq\left|W^{K}\right|$, then $\left|V^{J}\right|>\left|V^{K}\right|$ and

$$
1+\left|V^{K}\right| \leq\left|V^{J}\right| \leq 1+c^{J} Y .
$$

This inequality fails to imply condition (b) of the isomorphism part of the suspension theorem (applied to $\pi_{V}^{G}(Y)$ ) only because of the 1 on the righthand side. Moreover, if all the gaps in the fixed point dimensions of $V$ are at least 2 , then the inequality above becomes

$$
1+\left|V^{K}\right| \leq-1+\left|V^{J}\right| \leq c^{J} Y,
$$

and condition (b) of the isomorphism part of the suspension theorem is automatically satisfied. We formalize these observations in the following definition and proposition.

Definition 3.5. (i) If $V$ is a $G$-representation, then let $V^{\infty}$ be the direct sum of countably many copies of $V$. Note that $V^{\infty}$ is a $G$-universe in the sense of $[15$, p. 11] whenever $V$ contains a copy of the trivial representation.

(ii) A $G$-representation $V$ is sufficiently large if $\left|V^{G}\right| \geq 2$ and, whenever $J<K \leq G$ and $\left|V^{J}\right|>\left|V^{K}\right|$, then $\left|V^{J}\right| \geq\left|V^{K}\right|+2$. Observe that if $V$ is a complex $G$-representation that contains a trivial representation, then $V$ is sufficiently large. Also, if $V$ is a $G$-representation containing at least two copies of each of its irreducible subrepresentations and containing a trivial representation, then $V$ is sufficiently large. In particular, if $V$ is the sum of two copies of a representation containing a trivial representation, then $V$ is sufficiently large.

Proposition 3.6. Let $V$ be a G-representation with $\left|V^{G}\right| \geq 2$ and $Y$ be a $(V-1)$-connected, based $G-C W$ complex. Let $W$ be a subrepresentation of $n V$, for some $n \geq 1$. Then the following assertions are true:

(i) If $1+\left|V^{K}\right| \leq c^{J} Y$ for every subgroup $K$ of $\operatorname{Iso}\left(S^{V}\right)$ and every subgroup $J$ of $K$ with $\left|V^{J}\right|>\left|V^{K}\right|$, then the maps

$$
\sigma: \pi_{V}^{G}(Y) \rightarrow \pi_{V+W}^{G}\left(\Sigma^{W} Y\right), \quad \sigma: \pi_{V}^{G}(Y) \rightarrow \pi_{V}^{G}\left(\Sigma^{\infty} Y\right)
$$

are isomorphisms, provided that the stable homotopy group $\pi_{V}^{G}\left(\Sigma^{\infty} Y\right)$ is computed with respect to the universe $V^{\infty}$.

(ii) If $V$ is sufficiently large, then both of the maps $\sigma$ in part (i) are isomorphisms.

Our observations about suspending $V$-homotopy groups by subrepresentations of $n V$ also suffice to prove the following result about the functors $s$ of Definition 1.4(iii).

Lemma 3.7. Let $V$ be a G-representation with $\left|V^{G}\right| \geq 2$. Then the functor

$$
s: \mathscr{B}_{G}(V) \rightarrow \mathscr{B}_{G}\left(V^{\infty}\right),
$$

is an epimorphism on morphism sets. Moreover, if $V$ is sufficiently large, then this functor $s$ is an isomorphism.

\section{The CATEgories $\mathscr{B}_{G}(V)$ AND THE FUNCtors $s^{*}$ AND $s_{*}$}

The remainder of this article focuses on cases where the suspension map

$$
\sigma: \pi_{V}^{G}(Y) \rightarrow \pi_{V+W}^{G}\left(\Sigma^{W} Y\right)
$$


and the stabilization map

$$
\sigma: \pi_{V}^{G}(Y) \rightarrow \pi_{V}^{G}\left(\Sigma^{\infty} Y\right)
$$

are not isomorphisms, but induce isomorphisms from a modification $s_{*} \pi_{V}^{G}(Y)$ of $\pi_{V}^{G}(Y)$ to $\pi_{V+W}^{G}\left(\Sigma^{W} Y\right)$ or $\pi_{V}^{G}\left(\Sigma^{\infty} Y\right)$. In this section, we give an explicit method for computing the functors $s_{*}$ and summarize the properties of both the functors $s_{*}$ and their adjoints $s^{*}$. To do this, we must first give an explicit description of the structure of the categories $\mathscr{B}_{G}(V)$.

The category $\mathscr{B}_{G}$ is, by definition, isomorphic to the full subcategory of the equivariant stable category whose objects are the suspension spectra $\Sigma^{\infty} G / K^{+}$ of the orbits of $G$. Recall from [15, $\S \mathrm{V} .9]$ that, for any two subgroups $K$ and $H$ of $G$, the set of morphisms in $\mathscr{B}_{G}$ from $G / K$ to $G / H$ is the free abelian group whose generators correspond, under the isomorphism, to composites of the form

$$
\Sigma^{\infty} G / K^{+} \stackrel{\tau}{\longrightarrow} \Sigma^{\infty} G / J^{+} \stackrel{\Sigma^{\infty} \rho^{+}}{\longrightarrow} \Sigma^{\infty} G / H^{+},
$$

where $J \leq K, \tau$ is the transfer associated to the equivariant bundle projection $q: G / J \rightarrow G / K$, and $\rho$ is a space-level $G$-map from $G / J$ to $G / H$. When $G$ is not finite, we must impose the restriction that the index of $J$ in its $K$ normalizer $N_{K} J$ is finite. If $J=K$, then the composite $\left(\Sigma^{\infty} \rho^{+}\right) \tau=\Sigma^{\infty} \rho^{+}$is called a restriction map; it is denoted by $\rho$.

The composite $\left(\Sigma^{\infty} \rho^{+}\right) \tau$ is equal to the composite

$$
\Sigma^{\infty} G / K^{+} \stackrel{\tau^{\prime}}{\longrightarrow} \Sigma^{\infty} G / L^{+} \stackrel{\Sigma^{\infty} \mu^{+}}{\longrightarrow} \Sigma^{\infty} G / H^{+}
$$

if and only if there is a $G$-homeomorphism $\alpha: G / J \rightarrow G / L$ such that $\rho$ is $G$-homotopic (on the space-level) to $\mu \alpha$ and the bundle projection

$$
q: G / J \rightarrow G / K
$$

is equal (on the space-level) to the composite of the bundle projection $r: G / L \rightarrow$ $G / K$ and the map $\alpha$.

Any $G$-map $\rho: G / J \rightarrow G / H$ is, of course, determined by the image of the identity coset $e J$ of $G / J$. If this image is the coset $g H$ of $G / H$, then $g^{-1} J g \leq H$. Moreover, $\rho$ is a $G$-homeomorphism if and only if $g^{-1} J g=H$. Hereafter, we denote the $G$-map $\rho$ such that $\rho(e J)=g H$ by $\hat{g}$.

The morphisms in the category $\mathscr{B}_{G}(V)$ may be computed using results from $[3,8]$. If $V$ is sufficiently large (in the sense of Definition 3.5(ii)), then the results of this computation have a particularly nice description.

Proposition 4.1. Let $V$ be a sufficiently large G-representation. Then the stabilization functor $s: \mathscr{B}_{G}(V) \rightarrow \mathscr{B}_{G}$ is injective on morphism sets. The set $\mathscr{B}_{G}(V)(G / K, G / H)$ of morphisms in $\mathscr{B}_{G}(V)$ from $G / K$ to $G / H$ is isomorphic to the subgroup of $\mathscr{B}_{G}(G / K, G / H)$ generated by the composites of the form

$$
\Sigma^{\infty} G / K^{+} \stackrel{\tau}{\longrightarrow} \Sigma^{\infty} G / J^{+} \stackrel{\Sigma^{\infty} \rho^{+}}{\longrightarrow} \Sigma^{\infty} G / H^{+}
$$

for which $K / J$ is embeddable as a $K$-space in $V$.

In order to describe the results of this computation when $V$ is not sufficiently large, we must reiterate the definition of the transfer maps (see Remarks IV.2.4(ii) and IV.3.3(i) of [15]), but without invoking any of the usual stability assumptions. 
Definition 4.2. (i) Let $V$ be a $G$-representation and let $J \leq K \leq G$. The Weyl group $N_{K} J / J$ of $J$ is denoted $W_{K} J$. Let $V_{J, K}$ be the subset of $V$ consisting of those points whose $K$-isotropy subgroup is $J$, and let $\pi V_{J, K}$ denote the set of path components of $V_{J, K}$. The points in $V_{J, K}$ are in a one-to-one correspondence with the $K$-equivariant embeddings of $K / J$ in $V$. The group $W_{K} J$ acts on both $V_{J, K}$ and $\pi V_{J, K}$. By Theorem IV.3.1 of [2] applied to the action of $W_{K} J$ on $V^{J}, W_{K} J$ acts transitively on $\pi V_{J, K}$.

(ii) We want to associate to each $x \in V_{J, K}$ an unstable transfer map

$$
\tau_{x}: \Sigma^{V} G / K^{+} \rightarrow \Sigma^{V} G / J^{+}
$$

Let $i_{x}: K / J \rightarrow V$ be the $K$-embedding of $K / J$ in $V$ sending $e J$ to $x$. The tangent space $L$ of $K / J$ at $e J$ is a $J$-subrepresentation of $V$. Let $N$ be its orthogonal complement. There is a $K$-equivariant neighborhood of $i_{x}(K / J)$ in $V$ of the form $K \times_{J} N$. Let $f: S^{V} \rightarrow K^{+} \wedge_{J} S^{N}$ be the $K$-map collapsing out the complement of this tubular neighborhood. The unstable pretransfer associated to $x$ is the composite

$$
\hat{\tau}_{x}: S^{V} \stackrel{f}{\longrightarrow} K^{+} \wedge_{J} S^{N} \rightarrow K^{+} \wedge_{J} S^{V} \cong K / J^{+} \wedge S^{V},
$$

in which the second map is induced by the inclusion of $S^{N}$ into $S^{V}$. The unstable transfer associated to $x$ is the composite

$$
\tau_{x}: \Sigma^{V} G / K^{+} \cong G^{+} \wedge_{K} S^{V} \stackrel{1 \wedge_{K} \hat{\imath}_{x}}{\longrightarrow} G^{+} \wedge_{K}\left(K / J^{+} \wedge S^{V}\right) \cong \Sigma^{V} G / J^{+} .
$$

If the index of $J$ in $N_{K} J$ is not finite, then this map will be $G$-nullhomotopic.

The unstable transfers $\tau_{x}$ and $\tau_{y}$ coming from two points in $V_{J, K}$ are $G$ homotopic if and only if $x$ and $y$ are in the same path component of $V_{J, K}$. The real significance of $V$ being sufficiently large is that then, for each pair $J$ and $K$, the set $V_{J, K}$ is path connected, so there is, up to $G$-homotopy, a unique unstable transfer from $\Sigma^{V} G / K^{+}$to $\Sigma^{V} G / J^{+}$. The multiplicity of unstable transfers (indexed on $\pi V_{J, K}$ ) is the phenomenon which distinguishes the morphism sets of $\mathscr{B}_{G}(V)$ for nonsufficiently large $V$ from those for sufficiently large $V$. The action of $N_{K} J$ on $V_{J, K}$ provides a simple mechanism for keeping track of these multiple transfers. Each $g$ in $N_{K} J$ induces a $G$ homeomorphism $\hat{g}: G / J \rightarrow G / J$ such that, for each $x \in V_{J, K}$,

$$
\left(\Sigma^{V}\left(\hat{g}^{-1}\right)^{+}\right) \tau_{x}=\tau_{g x} .
$$

Thus, $N_{K} J$ acts transitively, by composition, on the set of $G$-homotopy classes of unstable transfers.

Proposition 4.3. Let $V$ be any G-representation with $\left|V^{G}\right| \geq 2$. The set $\left[\Sigma^{V} G / K^{+}, \Sigma^{V} G / H^{+}\right]_{G}$, which is the set of morphisms in $\mathscr{B}_{G}(V)$ from $G / K$ to $G / H$, is a free abelian group whose generators are equivalence classes of composites of the form

$$
\Sigma^{V} G / K^{+} \stackrel{\tau_{x}}{\longrightarrow} \Sigma^{V} G / J^{+} \stackrel{\Sigma^{V} \rho^{+}}{\longrightarrow} \Sigma^{V} G / H^{+},
$$

where $\rho: G / J \rightarrow G / H$ is a G-map, $J$ has finite index in $N_{K} J$, and $\tau_{x}$ is the transfer associated to a point $x$ in $V_{J, K}$. This composite is equal to the composite

$$
\Sigma^{V} G / K^{+} \stackrel{\tau_{y}}{\longrightarrow} \Sigma^{V} G / L^{+} \stackrel{\Sigma^{V} \mu^{+}}{\longrightarrow} \Sigma^{V} G / H^{+}
$$


if and only if there is a G-homeomorphism $\hat{g}: G / J \rightarrow G / L$ such that the following hold:

(a) $\rho$ is G-homotopic (on the space-level) to $\mu \hat{g}$,

(b) the bundle projection $q: G / J \rightarrow G / K$ is equal (on the space-level) to the composite of the bundle projection $r: G / L \rightarrow G / K$ and the map $\hat{g}$, and

(c) $x$ and $g y$ are in the same path component of $V_{J, K}$.

Example 4.4. Let $G$ be the group $\mathbb{Z} / 2, W$ be the real one-dimensional sign representation of $G$, and $V$ be $3+W$. The morphism sets of $\mathscr{B}_{G}(V)$ are, of course, described by the proposition, but they may also be obtained easily using the cofiber sequence

$$
G^{+} \cong S W^{+} \rightarrow D W^{+} \simeq S^{0} \rightarrow S^{W} .
$$

The direct computation has the advantage that it clearly displays the connection between the path components of $V_{\{e\}, G}$ and the corresponding unstable transfers. The results of the computation are:

$$
\begin{array}{ll}
\mathscr{B}_{G}(V)(G /\{e\}, G /\{e\})=\mathbb{Z} \oplus \mathbb{Z}, & \mathscr{B}_{G}(V)(G /\{e\}, G / G)=\mathbb{Z}, \\
\mathscr{B}_{G}(V)(G / G, G /\{e\})=\mathbb{Z} \oplus \mathbb{Z}, & \mathscr{B}_{G}(V)(G / G, G / G)=\mathbb{Z} \oplus \mathbb{Z} .
\end{array}
$$

The suspension functor $s: \mathscr{B}_{G}(V) \rightarrow \mathscr{B}_{G}$ maps each of these morphism sets except $\mathscr{B}_{G}(V)(G / G, G /\{e\})$ isomorphically to the corresponding morphism set for $\mathscr{B}_{G}$. The two unstable transfers which generate $\mathscr{B}_{G}(V)(G / G, G /\{e\})$ are identified to a single stable transfer by this suspension functor. Other examples of multiple transfers may be obtained by taking $G$ to be a dihedral group or $\mathrm{O}(2)$ and $W$ to be the obvious 2-dimensional irreducible real $G$-representation.

If $V$ and $W$ are $G$-representations with $W \subset V$ and $\left|W^{G}\right| \geq 2$, then the categories $\mathscr{M}_{G}(V)$ and $\mathscr{M}_{G}(W)$ and the functor $s^{*}: \mathscr{M}_{G}(V) \rightarrow \mathscr{M}_{G}(W)$ are nicely behaved in almost every possible way. The category $\mathscr{M}_{G}(V)$, being a category of additive functors into the category of abelian groups, is an abelian category satisfying all the axioms needed for homological algebra [6, 20]. All small limits and colimits exist in $\mathscr{M}_{G}(V)$ and are computed termwise. A morphism (natural transformation) $f: M \rightarrow N$ in $\mathscr{M}_{G}(V)$ is an epimorphism, monomorphism, or isomorphism if and only if all of its values $f(G / H): M(G / H) \rightarrow N(G / H)$ are such. The representable functors in $\mathscr{M}_{G}(V)$ are just the $V$-Mackey functors $\pi_{V}^{G}\left(\Sigma^{V} G / K^{+}\right)$; these functors are projective in $\mathscr{M}_{G}(V)$. The functor $s^{*}: \mathscr{M}_{G}(V) \rightarrow \mathscr{M}_{G}(W)$, being a forgetful functor, is the identity on both objects and morphisms. It identifies $\mathscr{M}_{G}(V)$ with a subcategory of $\mathscr{M}_{G}(W)$. If $s: \mathscr{B}_{G}(W) \rightarrow \mathscr{B}_{G}(V)$ is an epimorphism on morphism sets, then so is $s^{*}$. In this case, $\mathscr{M}_{G}(V)$ is isomorphic to a full subcategory of $\mathscr{M}_{G}(W)$. In any case, $s^{*}$ preserves and reflects all limits, colimits, monomorphisms, and epimorphisms.

The functors $s_{*}$ are not so nicely behaved. We begin with their formal definition and the properties that follow trivially from it.

Lemma 4.5. Let $W \subset V \subset U$ be G-representations with $\left|W^{G}\right| \geq 2$ and let

$$
s: \mathscr{B}_{G}(W) \rightarrow \mathscr{B}_{G}(V), \quad s^{\prime}: \mathscr{B}_{G}(V) \rightarrow \mathscr{B}_{G}(U)
$$

be the associated suspension functors. Then the following assertions are true: 
(i) The functor

has a left adjoint

$$
s^{*}: \mathscr{M}_{G}(V) \rightarrow \mathscr{M}_{G}(W)
$$

$$
s_{*}: \mathscr{M}_{G}(W) \rightarrow \mathscr{M}_{G}(V) .
$$

Being a left adjoint, $s_{*}$ is right exact and preserves all colimits.

(ii) The functor $s_{*}$ preserves representable functors in the sense that the suspension map

$$
\tilde{\sigma}: s_{*} \pi_{W}^{G}\left(\Sigma^{W} G / K^{+}\right) \rightarrow \pi_{V}^{G}\left(\Sigma^{V} G / K^{+}\right)
$$

is an isomorphism.

(iii) If the functor $s: \mathscr{B}_{G}(W) \rightarrow \mathscr{B}_{G}(V)$ is an epimorphism on morphism sets, then, for any $V$-Mackey functor $M$, the counit $\varepsilon: s_{*} s^{*} M \rightarrow M$ of the $\left(s_{*}, s^{*}\right)$ adjunction is an isomorphism.

(iv) Let $M \in \mathscr{M}_{G}(V)$ and $N \in \mathscr{M}_{G}(W)$. Let $f: N \rightarrow s^{*} M$ be a morphism in $\mathscr{M}_{G}(W)$ and let $\tilde{f}: s_{*} N \rightarrow M$ be its adjoint. If the component $f(G / K): N(G / K) \rightarrow\left(s^{*} M\right)(G / K)$ of $f$ at an orbit $G / K$ is an epimorphism, then so is the component $\tilde{f}(G / K)$ of $\tilde{f}$. If $s: \mathscr{B}_{G}(W) \rightarrow \mathscr{B}_{G}(V)$ is an epimorphism on morphism sets and $f$ is an isomorphism, then $\tilde{f}$ is also an isomorphism.

(v) The functors $\left(s^{\prime} s\right)_{*}$ and $\left(s^{\prime}\right)_{*} s_{*}$ are naturally isomorphic.

Proof. Since $\mathscr{M}_{G}(W)$ and $\mathscr{M}_{G}(V)$ are functor categories and $s^{*}$ is given by precomposition, the left adjoint is given by left Kan extension [17, Corollary 2, p. 235]. As a left adjoint functor between abelian categories, $s_{*}$ must be right exact and preserve all colimits. The preservation of representables follows from an easy Yoneda lemma argument. Part (iii) follows from [17, Theorem 1, p. 88] since $s^{*}$ is full and faithful. For the epimorphism assertion in the fourth part, consider the diagram

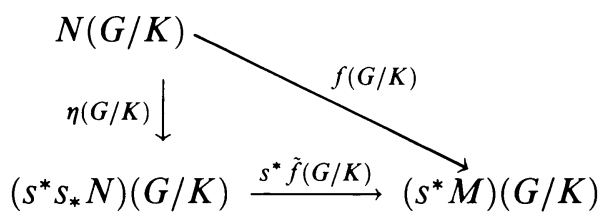

in which $\eta$ is the unit of the $\left(s_{*}, s^{*}\right)$ adjunction. If $f(G / K)$ is an epimorphism, then so is $s^{*} \tilde{f}(G / K)$. But as a homomorphism of abelian groups, $s^{*} \tilde{f}(G / K)$ is just $\tilde{f}(G / K)$. Thus, $\tilde{f}(G / K)$ is an epimorphism. For the isomorphism assertion in (iv), apply (iii) and the observation that $\tilde{f}$ is the composite

$$
s_{*} N \stackrel{s_{*} f}{\longrightarrow} S_{*} s^{*} M \stackrel{\varepsilon}{\longrightarrow} M,
$$

where $\varepsilon$ is the counit of the adjunction. Part (v) follows by adjunction from the obvious equality of $\left(s^{\prime} s\right)^{*}$ and $s^{*}\left(s^{\prime}\right)^{*}$.

Warning 4.6. The functor $s_{*}: \mathscr{M}_{G}(W) \rightarrow \mathscr{M}_{G}(V)$ is not exact unless

$$
s: \mathscr{B}_{G}(W) \rightarrow \mathscr{B}_{G}(V)
$$

is an isomorphism (so that $s_{*}$ is also an isomorphism).

Arguments about equivariant homotopy groups which employ induction over the subgroups of $G$ often rely heavily on the fact that the value of. $\pi_{W}^{G}(Y)$ at the 
orbit $G / H$ is just $\pi_{W}^{H}(Y)$, which depends only on $H$ and not on the ambient group $G$. It is very important for our inductive arguments in later sections that the functor $s_{*} \pi_{W}^{G}(Y)$ have a similar property. If $G$ is a finite group, then this aspect of the behavior of $s_{*}$ can be derived formally from a dual property of the adjoint functor $s^{*}$. For arbitrary compact Lie groups, this property must be derived from the construction of $s_{*}$. Thus we state this property precisely now, but delay its proof until after the construction of $s_{*}$.

For any subgroup $H$ of $G$ and any $G$-representation $V$, there is a functor $r_{V}: \mathscr{B}_{H}(V) \rightarrow \mathscr{B}_{G}(V)$ which takes the $H$-orbit $H / K$ to the $G$-orbit $G / K$ and the $H$-map $f: \Sigma^{V} H / J^{+} \rightarrow \Sigma^{V} H / K^{+}$to the composite

$$
\Sigma^{V} G / J^{+} \cong G^{+} \wedge_{H} \Sigma^{V} H / J^{+} \stackrel{G^{+} \wedge_{H} f}{\longrightarrow} G^{+} \wedge_{H} \Sigma^{V} H / K^{+} \cong \Sigma^{V} G / K^{+} .
$$

The functor $r_{V}$ induces a functor

$$
r_{V}^{*}: \mathscr{M}_{G}(V) \rightarrow \mathscr{M}_{H}(V)
$$

that restricts $G-V$-Mackey functors down to $H-V$-Mackey functors. As an example of the effect of $r_{V}^{*}$, notice that, for any $G$-space $Y, r_{V}^{*} \pi_{V}^{G}(Y)$ is just $\boldsymbol{\pi}_{V}^{H}(Y)$. Let

$$
s_{*}^{G}: \mathscr{M}_{G}(W) \rightarrow \mathscr{M}_{G}(V), \quad s_{*}^{H}: \mathscr{M}_{H}(W) \rightarrow \mathscr{M}_{H}(V)
$$

be the functors associated to the inclusion of $W$ into $V$ as a $G$-representation. Precisely stated, the property of the functors $S_{*}$ which is critical for induction arguments is their relation to the functors $r^{*}$.

Proposition 4.7. Let $H$ be a subgroup of $G$ and let $W \subset V$ be G-representations with $\left|W^{G}\right| \geq 2$. Then the composite functors $r_{V}^{*} s_{*}^{G}$ and $s_{*}^{H} r_{W}^{*}$ from $\mathscr{M}_{G}(W)$ to $\mathscr{M}_{H}(V)$ are naturally isomorphic.

The intuition behind our construction of the functor $s_{*}$ associated to the inclusion of $W$ in $V$ is very straightforward. The two possible differences between the categories $\mathscr{B}_{G}(W)$ and $\mathscr{B}_{G}(V)$ are that $\mathscr{B}_{G}(V)$ may contain some transfers that are not in $\mathscr{B}_{G}(W)$ and that, if $W$ is not sufficiently large, then there may be multiple transfers in $\mathscr{B}_{G}(W)$ from $G / K$ to $G / H$ which are identified to a single transfer in $\mathscr{B}_{G}(V)$. Let $N$ be a $W$-Mackey functor. To allow for a transfer $\tau$ from $G / K$ to $G / H$ which appears in $\mathscr{B}_{G}(V)$, but not in $\mathscr{B}_{G}(W)$, we must provide each element of $N(G / H)$ with an image under $\tau$ in $\left(s_{*} N\right)(G / K)$. To cope with the identification of multiple transfers from $\mathscr{B}_{G}(W)$ into a single transfer in $\mathscr{B}_{G}(V)$, we must impose relations on $\left(s_{*} N\right)(G / K)$ identifying the images of each element of $N(G / H)$ under the multiple transfers.

The description of the behavior of $s_{*} N$ on the morphisms of $\mathscr{B}_{G}(V)$ is simplified by the fact that every map in $\mathscr{B}_{G}(V)$ is the composite of a transfer and the suspension by $V$ of some $G$-map $\hat{g}: G / K_{1} \rightarrow G / K_{2}$. Thus, it suffices to describe the behavior of $s_{*} N$ with respect to transfers and the maps coming from the $\hat{g}$.

Full details of this construction are given only for the special case in which $V$ is sufficiently large. The modifications needed to handle the general case are then sketched in Remarks 4.9(iii). 
Construction 4.8. Let $W \subset V$ be $G$-representations with $\left|W^{G}\right| \geq 2$ and $V$ sufficiently large. Let $N$ be a $W$-Mackey functor and $s: \mathscr{B}_{G}(W) \rightarrow \mathscr{B}_{G}(V)$ be the suspension functor.

(i) Let $K \leq G$. Then

$$
\left(s_{*} N\right)(G / K)=\operatorname{coker}\left(\Theta_{1}-\Theta_{2}\right),
$$

for two maps $\Theta_{1}$ and $\Theta_{2}$. Let $\widetilde{\Gamma}(K)$ be the set of subgroups $J$ of $K$ such that $W_{K} J$ is finite and $K / J$ embeds as a $K$-space in $V$. Let $\Gamma(K)$ be a subset of $\widetilde{\Gamma}(K)$ consisting of one representative of each $K$-conjugacy class of subgroups in $\widetilde{\Gamma}(K)$. Notice that $K$ is in $\Gamma(K)$. Let $\Lambda(K)$ be the set of quadruples $\left(g, H, J_{1}, J_{2}\right)$ with $g \in K, H \leq K$, and $J_{1}, J_{2} \in \Gamma(K)$ such that:

(a) $H<J_{1}$ and $J_{1} / H$ embeds in $W$ as a $J_{1}$-space, and

(b) $g \mathrm{Hg}^{-1} \leq J_{2}$ and $J_{2} /\left(g \mathrm{Hg}^{-1}\right)$ embeds in $W$ as a $J_{2}$-space.

For each pair of subgroups $H \leq J$ in $G$ such that $J / H$ embeds in $W$ as a $J$-space, pick a transfer $\tau(H, J)$ from $G / J$ to $G / H$ in $\mathscr{B}_{G}(W)$. Define the map

$$
\Theta_{1}: \sum_{\left(g, H, J_{1}, J_{2}\right) \in \Lambda(K)} N(G / H) \rightarrow \sum_{J \in \Gamma(K)} N(G / J) / W_{K} J
$$

by requiring that its restriction to the summand $N(G / H)$ of its domain indexed on $\left(g, H, J_{1}, J_{2}\right) \in \Lambda(K)$ be the map

$$
N(G / H) \stackrel{N\left(\tau\left(H, J_{1}\right)\right)}{\longrightarrow} N\left(G / J_{1}\right) / W_{K} J_{1} \subset \sum_{J \in \Gamma(K)} N(G / J) / W_{K} J .
$$

The restriction of the map

$$
\Theta_{2}: \sum_{\left(g, H, J_{1}, J_{2}\right) \in \Lambda(K)} N(G / H) \rightarrow \sum_{J \in \Gamma(K)} N(G / J) / W_{K} J
$$

to the summand $N(G / H)$ of its domain indexed on $\left(g, H, J_{1}, J_{2}\right) \in \Lambda(K)$ is 0 if $W_{K} H$ is not finite. Otherwise, it is the map

$$
N(G / H) \stackrel{N(\hat{g} \tau)}{\longrightarrow} N\left(G / J_{2}\right) / W_{K} J_{2} \subset \sum_{J \in \Gamma(K)} N(G / J) / W_{K} J,
$$

where $\tau=\tau\left(g H g^{-1}, J_{2}\right)$.

(ii) To describe the behavior of $s_{*} N$ on the transfer $\tau$ from $G / K_{2}$ to $G / K_{1}$ in $\mathscr{B}_{G}(V)$, we construct a map

$$
\Phi: \sum_{J_{1} \in \Gamma\left(K_{1}\right)} N\left(G / J_{1}\right) / W_{K_{1}} J_{1} \rightarrow \sum_{J_{2} \in \Gamma\left(K_{2}\right)} N\left(G / J_{2}\right) / W_{K_{2}} J_{2}
$$

which induces the map $\left(s_{*} N\right)(\tau):\left(s_{*} N\right)\left(G / K_{1}\right) \rightarrow\left(s_{*} N\right)\left(G / K_{2}\right)$ on the cokernels. Let $J_{1}$ be an element of the indexing set $\Gamma\left(K_{1}\right)$. If $W_{K_{2}} J_{1}$ is not finite, then the restriction of $\Phi$ to the summand indexed on $J_{1}$ is 0 . Assume $W_{K_{2}} J_{1}$ is finite. Since $K_{1} / J_{1}$ embeds in $V$ as a $K_{1}$-space and $K_{2} / K_{1}$ embeds in $V$ as a $K_{2}$-space, $K_{2} / J_{1}$ embeds in $V$ as a $K_{2}$-space. Thus, a $K_{2}$-conjugate $J_{2}$ of $J_{1}$ is in $\Gamma\left(K_{2}\right)$. Pick $g \in K_{2}$ such that $g J_{1} g^{-1}=J_{2}$. Define $\Phi$ on the summand of its domain indexed by $J_{1}$ to be the composite

$$
N\left(G / J_{1}\right) / W_{K_{1}} J_{1} \stackrel{N(\hat{g})}{\longrightarrow} N\left(G / J_{2}\right) / W_{K_{2}} J_{2} \subset \sum_{J_{2} \in \Gamma\left(K_{2}\right)} N\left(G / J_{2}\right) / W_{K_{2}} J_{2} .
$$


(iii) To describe the behavior of $s_{*} N$ on the restriction $\hat{g}: G / K_{1} \rightarrow G / K_{2}$ in $\mathscr{B}_{G}(V)$, we construct a map

$$
\Delta: \sum_{H \in \Gamma\left(K_{2}\right)} N(G / H) / W_{K_{2}} H \rightarrow \sum_{J \in \Gamma\left(K_{1}\right)} N(G / J) / W_{K_{1}} J
$$

which induces the map $\left(s_{*} N\right)(\hat{g}):\left(s_{*} N\right)\left(G / K_{2}\right) \rightarrow\left(s_{*} N\right)\left(G / K_{1}\right)$ on the cokernels. Let $H$ be an element of the indexing set $\Gamma\left(K_{2}\right)$ and

$$
\tau: \Sigma^{\infty} G / K_{2}^{+} \rightarrow \Sigma^{\infty} G / H^{+}
$$

be the transfer in $\mathscr{B}_{G}$. The double coset formula provides a decomposition

$$
\tau \Sigma^{\infty} \hat{g}^{+}=\sum_{i=1}^{m} n_{i} \hat{g}_{i}^{+} \tau_{i}
$$

where the $\tau_{i}: \Sigma^{\infty} G / K_{1}^{+} \rightarrow \Sigma^{\infty} G / J_{i}^{+}$are the transfers associated to certain subgroups $J_{i}$ of $K_{1}$, the $g_{i}$ are elements of $G$ inducing $G$-maps

$$
\hat{g}_{i}: G / J_{i} \rightarrow G / H,
$$

and the $n_{i}$ are integers. For every $i$, the orbit $K_{1} / J_{i}$ embeds as a $K_{1}$-space in $V$ since $K_{2} / H$ embeds as a $K_{2}$-space in $V$ and the subgroup $J_{i}$ is the intersection of $K_{1}$ with some conjugate of $H$. Moreover, for each $i, W_{K_{1}} J_{i}$ is finite. Thus, some conjugate of $J_{i}$ must be in $\Gamma\left(K_{1}\right)$. By adjusting the $g_{i}$, we may assume that each $J_{i}$ is in $\Gamma\left(K_{1}\right)$. The restriction of $\Delta$ to the summand of its domain indexed on $H$ is then the composite

$$
N(G / H) / W_{K_{2}} H \stackrel{\sum n_{i} N\left(\hat{g}_{i}\right)}{\longrightarrow} \sum_{i=1}^{n} N\left(G / J_{i}\right) / W_{K_{1}} J_{i} \subset \sum_{J \in \Gamma\left(K_{1}\right)} N(G / J) / W_{K_{1}} J .
$$

Remarks 4.9. (i) Let $N$ be a $W$-Mackey functor. Since $s_{*}$ is the left adjoint to $s^{*}$, the functor $s_{*} N$ is a left Kan extension. Thus, its value at an orbit $G / K$ is

$$
\left(s_{*} N\right)(G / K)=\int^{G / J} \mathscr{B}_{G}(V)(G / K, G / J) \otimes N(G / J),
$$

where the coend is taken over the category $\mathscr{B}_{G}(W)[17$, Theorem 1, p. 236]. To verify that Construction 4.8 actually produces $\left(s_{*} N\right)(G / K)$, it suffices to show that it produces the coend. This verification is not difficult since the morphism sets $\mathscr{B}_{G}(V)(G / K, G / J)$ are free abelian groups.

(ii) The indexing sets for the sums giving the domain and range of the maps $\Theta_{i}$ are, unfortunately, rather large. In some cases, they may be trimmed. An element $J$ in the indexing set $\Gamma(K)$ of the construction really should be regarded as representing the corresponding transfer $\tau: G / K \rightarrow G / J$ in $\mathscr{B}_{G}(V)$. If this transfer factors as a composite

$$
G / K \stackrel{\tau_{1}}{\longrightarrow} G / L \stackrel{\tau_{2}}{\longrightarrow} G / J
$$

with $J<L, \tau_{1}$ in $\mathscr{B}_{G}(V)$, and $\tau_{2}$ in $\mathscr{B}_{G}(W)$, then deleting the single element $J$ from $\Gamma(K)$ and the associated elements from $\Lambda(K)$ does not affect the cokernel of $\Theta_{1}-\Theta_{2}$. If $G$ is finite, then all of these extraneous elements of $\Gamma(K)$ and $\Lambda(K)$ may be deleted simultaneously without affecting the cokernel. This can significantly simplify the description of $s_{*}$. If $G$ is not finite, then 
there may be infinite increasing sequences of these extraneous subgroups. The cokernel will be unchanged if any finite subset of such a sequence is deleted, but it may be changed by deleting the whole sequence.

(iii) Three changes must be made to our description of $\left(s_{*} N\right)(G / K)$ if $V$ is not sufficiently large. Let $J$ be an element of $\Gamma(K)$ and select a path component $P$ of $V_{J, K}$. Let $W_{K} P$ be the subgroup of $W_{K} J$ that fixes $P$ in the action of $W_{K} J$ on $\pi V_{J, K}$. The first change is that the $J$-indexed summand $N(G / J) / W_{K} J$ of the range of the $\Theta_{i}$ must be replaced by $N(G / J) / W_{K} P$.

To understand the second change, think of each element $\left(g, H, J_{1}, J_{2}\right)$ of $\Lambda(K)$ as a commuting diagram of the form

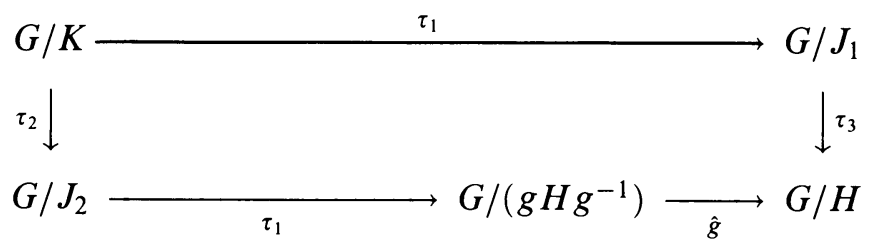

in $\mathscr{B}_{G}(V)$, where the transfers $\tau_{3}$ and $\tau_{4}$ are required to be the suspensions of transfers in $\mathscr{B}_{G}(W)$. If $V$ is sufficiently large and $W$ is not sufficiently large, then the various possible choices of the element $g$ account for the effect on $\left(s_{*} N\right)(G / K)$ of any collapsing of several transfers in $\mathscr{B}_{G}(W)$ into one transfer in $\mathscr{B}_{G}(V)$. If $V$ is not sufficiently large, then there may be several choices in $\mathscr{B}_{G}(V)$ for each of the transfers that appear in this diagram. The second change in the definition of $s_{*} N$ is that the indexing set $\Lambda(K)$ for the domain of the $\Theta_{i}$ should be the set of all commuting diagrams in $\mathscr{B}_{G}(V)$ of the form above, subject to the additional condition that the transfers $\tau_{1}$ and $\tau_{2}$ must correspond to the path components of $V_{J_{1}, K}$ and $V_{J_{2}, K}$ selected as a part of the first change. The third change is that the transfers $\tau\left(H, J_{1}\right)$ and $\tau\left(g \mathrm{Hg}^{-1}, J_{2}\right)$ used in defining the maps $\Theta_{1}$ and $\Theta_{2}$ on each summand of their domain should be preimages in $\mathscr{B}_{G}(W)$ of the transfers $\tau_{3}$ and $\tau_{4}$ appearing in the indexing diagram for that summand.

We conclude this section with the proofs of Propositions 4.7 and 1.9.

Proof of Proposition 4.7. Let $N$ be a $G$-W-Mackey functor and let $K$ be a subgroup of $H$. Observe that the construction of $\left(s_{*}^{G} N\right)(G / K)$ depends on the values of $N$ on the orbits $G / J$ associated to the subgroups $J$ of $K$, the behavior of $V$ as a $K$-representation, and the $K$-conjugacy classes of subgroups of $K$. Thus, the construction depends only on $K$ and not on the ambient group $G$. The abelian groups $\left(r_{V}^{*} s_{*}^{G} N\right)(G / K)$ and $\left(s_{*}^{H} r_{W}^{*} N\right)(G / K)$ are therefore identical. The same observation applies to the construction of $\left(s_{*}^{G} N\right)(f)$ for any map $f: G / J \rightarrow G / K$ in the image of the functor $r_{V}$.

Proof of Proposition 1.9. Assume that $N(G / J)=0$ for every proper subgroup $J$ of $K$. Then all of the summands in the domain of the maps $\Theta_{1}$ and $\theta_{2}$ used to define $\left(s_{*} N\right)(G / K)$ vanish. The only summand of the range of these maps that need not vanish is $N(G / K)$. Thus, the cokernel must be $N(G / K)$. If $N \neq 0$, then using the descending chain condition on the subgroups of $G$, it is possible to pick a minimal subgroup $K$ of $G$ on which $N$ is nonzero. By the first part of the proposition,

$$
\left(s_{*} N\right)(G / K)=N(G / K) \neq 0 .
$$




\section{A LOW-DIMENSIONAL SUSPENSION THEOREM}

Propositions 3.6 and 6.1 and the stable Hurewicz theorem suffice to prove a variant of the second version of the unstable Hurewicz theorem applicable only to representations that are sufficiently large in the sense of Definition 3.5(ii). A more specialized suspension theorem is needed to remove this restriction. Let $V$ be a $G$-representation with $\left|V^{G}\right| \geq 2$ and let $Y$ be a based $G$-space. Let

$$
s_{V}^{*}: \mathscr{M}_{G}(2 V) \rightarrow \mathscr{M}_{G}(V), \quad s_{*}^{V}: \mathscr{M}_{G}(V) \rightarrow \mathscr{M}_{G}(2 V)
$$

be the functors associated to the inclusion of $V$ into $2 V$ as the first summand. The suspension map induces a natural transformation

$$
\sigma_{V}: \pi_{V}^{G}(Y) \rightarrow s_{V}^{*} \pi_{2 V}^{G}\left(\Sigma^{V} Y\right)
$$

of $V$-Mackey functors and there is an adjoint natural transformation

$$
\tilde{\sigma}_{V}: s_{*}^{V} \pi_{V}^{G}(Y) \rightarrow \pi_{2 V}^{G}\left(\Sigma^{V} Y\right) \text {. }
$$

Our more specialized suspension theorem gives conditions under which $\tilde{\sigma}_{V}$ is an isomorphism.

Theorem 5.1. Let $V$ be a G-representation with $\left|V^{G}\right| \geq 2$ and let $Y$ be a based, $(V-1)$-connected $G$-CW complex. Then

$$
\tilde{\sigma}_{V}: s_{*}^{V} \pi_{V}^{G}(Y) \rightarrow \pi_{2 V}^{G}\left(\Sigma^{V} Y\right)
$$

is an isomorphism.

Proof. If $G$ is the trivial group, the theorem follows from the nonequivariant suspension theorem. Thus, we may prove the theorem by induction over the subgroups of $G$.

Let $W$ be the orthogonal complement of $V^{G}$ in $V$ and let $r=\left|V^{G}\right|$. If $W=0$, then our theorem follows from Namboodiri's result. Thus, we assume that $W \neq 0$. The map $\tilde{\sigma}_{V}$ is the composite

$$
\tilde{\sigma}_{V}: s_{*}^{V} \pi_{V}^{G}(Y) \cong s_{*}^{r} s_{*}^{W} \pi_{V}^{G}(Y) \stackrel{s_{*}^{r} \tilde{\sigma}_{W}}{\longrightarrow} s_{*}^{r} \pi_{V+W}^{G}\left(\Sigma^{W} Y\right) \stackrel{\tilde{\sigma}_{r}}{\longrightarrow} \pi_{2 V}^{G}\left(\Sigma^{V} Y\right),
$$

where the maps $\tilde{\sigma}_{W}$ and $\tilde{\sigma}_{r}$ are the obvious adjoint suspension maps and $s_{*}^{W}$ and $s_{*}^{r}$ are the functors associated to the inclusions of $V$ into $V+W$ and $V+W$ into $2 V$, respectively. Since the map $\tilde{\sigma}_{r}$ is an isomorphism by Namboodiri's theorem, to prove that $\tilde{\sigma}_{V}$ is an isomorphism, it suffices to prove that

$$
\tilde{\sigma}_{W}: s_{*}^{W} \boldsymbol{\pi}_{V}^{G}(Y) \rightarrow \boldsymbol{\pi}_{V+W}^{G}\left(\Sigma^{W} Y\right)
$$

is an isomorphism. Proposition 4.7 and our inductive hypothesis, that the theorem holds for all proper subgroups of $G$, allow us to assume that

$$
\tilde{\sigma}_{W}(G / K): s_{*}^{W} \pi_{V}^{G}(Y)(G / K) \rightarrow \pi_{V+W}^{G}\left(\Sigma^{W} Y\right)(G / K)
$$

is an isomorphism for every proper subgroup $K$ of $G$.

Our assumptions on $\left|V^{*}\right|$ and $c^{*} Y$ imply that $c^{*} Y \geq 1^{*}$. However, $\pi_{V}^{G}(Y)$ is stable with respect to suspension by the trivial representation. Thus, we may assume, by replacing $V$ by $V+1$ and $Y$ by $\Sigma Y$ if necessary, that $c^{*} Y \geq 2^{*}$.

We will prove that

$$
\tilde{\sigma}_{W}: s_{*}^{W} \boldsymbol{\pi}_{V}^{G}(Y) \rightarrow \boldsymbol{\pi}_{V+W}^{G}\left(\Sigma^{W} Y\right)
$$


is an isomorphism by using a second induction to prove that, for every subgroup $H$ in $\operatorname{Iso}\left(W^{\infty}\right)$, the map

$$
\tilde{\sigma}_{W}^{H}: s_{*}^{W} \pi_{V}^{G}\left(G / H^{+} \wedge Y\right) \rightarrow \pi_{V+W}^{G}\left(\Sigma^{W} G / H^{+} \wedge Y\right)
$$

is an isomorphism. Taking $H=G$ then gives that $\tilde{\sigma}_{W}=\tilde{\sigma}_{W}^{G}$ is an isomorphism. Let $L$ be the tangent space of $G / H$ at $e H$ regarded as an $H$-representation ( $L=0$ if $G / H$ is finite). The proof that $\tilde{\sigma}_{W}^{H}$ is an isomorphism breaks into three cases, depending on whether or not $W$ is trivial as an $H$-representation, and if $W$ is not $H$-trivial, on whether or not $L^{H}$ is zero. The first case, that $W$ is trivial as an $H$-representation, applies whenever $H$ is a minimal subgroup in Iso $\left(W^{\infty}\right)$. Thus, in the other two cases, we may employ our second inductive assumption, which is that $\tilde{\sigma}_{W}^{J}$ is an isomorphism for every proper subgroup $J$ of $H$ in $\operatorname{Iso}\left(W^{\infty}\right)$.

For any subgroup $H$ of $G$, the $G$-space $G / H^{+} \wedge Y$ is $G$-homeomorphic to $G^{+} \wedge_{H} Y$, the free $G$-space generated by $Y$ considered as an $H$-space. We may assume (if necessary, by replacing $Y$ by a based $H$-homotopy equivalent $H$ space) that $Y$ is a based $H$-CW complex with $H$-skeleton $Y^{q}$. Moreover, our connectivity hypothesis allows us to assume that the bottom $H$-cells of $Y$ are in a dimension no lower than $m=\left|V^{H}\right|$. The $H$-CW structure of $Y$ induces a $G$-CW structure on $G^{+} \wedge_{H} Y$ with $G$-skeleton $G^{+} \wedge_{H} Y^{q}$.

Now assume that $H \in \operatorname{Iso}\left(W^{\infty}\right)$ and that $W$ is trivial as a $H$-representation. By Namboodiri's theorem, $\pi_{V}^{G}\left(G^{+} \wedge_{H} Y^{q}\right)$ and $\pi_{V}^{G}\left(G^{+} \wedge_{H} Y\right)$ are stable with respect to the trivial representation. Thus, we may argue inductively on the $H$-skeleton of $Y$ to show that $\pi_{V}^{G}\left(G^{+} \wedge_{H} Y\right)$ is isomorphic to $\pi_{V}^{G}\left(G^{+} \wedge_{H} Y^{m+1}\right)$. Similarly, $\pi_{V+W}^{G}\left(\Sigma^{W} G^{+} \wedge_{H} Y\right)$ is isomorphic to $\pi_{V+W}^{G}\left(\Sigma^{W} G^{+} \wedge_{H} Y^{m+1}\right)$. The $H$-m-skeleton $Y^{m}$ of $Y$ is $\bigvee_{i}\left(H / K_{i}\right)^{+} \wedge S^{m}$ for some collection $K_{i}$ of subgroups of $H$. The $H$ - $(m+1)$-skeleton $Y^{m+1}$ is obtained from $Y^{m}$ by attaching a collection $\left\{\left(H / J_{j}\right)^{+} \wedge e^{m+1}\right\}_{j}$ of $(m+1)$-cells along based attaching maps. Thus, there is an $H$-equivariant cofiber sequence

$$
\bigvee_{j}\left(H / J_{j}\right)^{+} \wedge S^{m} \rightarrow Y^{m} \rightarrow Y^{m+1} .
$$

This cofiber sequence induces the diagram

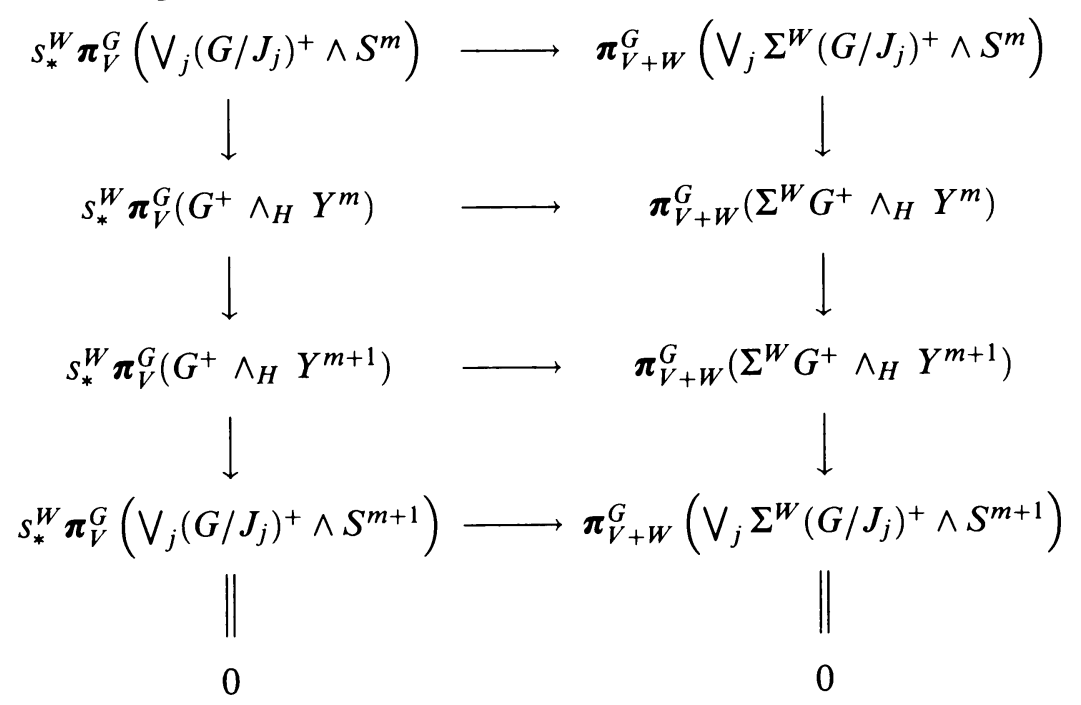


in which all the horizontal arrows are suspension maps. The morphism sets at the bottom of each column vanish by a simple connectivity argument since $m=\left|V^{H}\right|=|V|$. By Namboodiri's theorem, all of the morphism sets in the diagram are stable with respect to suspension by the trivial representation. Thus, the right column is exact, and the left column would be exact if the $s_{*}^{W}$ were omitted. Since the functor $s_{*}^{W}$, being a left adjoint, is right exact, the left column is exact. It follows by a diagram chase that $\tilde{\sigma}_{W}^{H}$ will be an isomorphism if

$$
\tilde{\sigma}_{W}^{J}: s_{*}^{W} \boldsymbol{\pi}_{V}^{G}\left(G / J^{+} \wedge S^{m}\right) \rightarrow \boldsymbol{\pi}_{V+W}^{G}\left(\Sigma^{W} G / J^{+} \wedge S^{m}\right)
$$

is an isomorphism for every subgroup $J$ of $H$. The space $G / J^{+} \wedge S^{m}$ may be replaced by $G / J^{+} \wedge S^{V}$ since the triviality of $W$ as an $H$-representation provides $G$-homeomorphisms

$$
G / J^{+} \wedge S^{m} \cong G^{+} \wedge_{J} S^{m} \cong G^{+} \wedge_{J} S^{V} \cong G / J^{+} \wedge S^{V}
$$

By Lemma 4.5(ii), the map

$$
\tilde{\sigma}_{W}^{J}: s_{*}^{W} \pi_{V}^{G}\left(G / H^{+} \wedge S^{V}\right) \rightarrow \pi_{V+W}^{G}\left(\Sigma^{W} G / J^{+} \wedge S^{V}\right)
$$

is an isomorphism.

Now assume that $W$ is not a trivial $H$-representation. In this situation, it is necessary to consider the maps

$$
\tilde{\sigma}_{n W}^{H}: s_{*}^{n W} \pi_{V}^{G}\left(G / H^{+} \wedge Y\right) \rightarrow \pi_{V+n W}^{G}\left(\Sigma^{n W} G / H^{+} \wedge Y\right)
$$

describing suspension by $n$ copies of $W$, for $n \geq 1$. By Proposition 3.6(ii), the functors

$$
s_{*}: \mathscr{M}_{G}(V+W) \rightarrow \mathscr{M}_{G}(V+n W)
$$

are isomorphisms. Also, by this result, $\pi_{V+W}^{G}\left(\Sigma^{W} G / H^{+} \wedge Y\right)$ is stable with respect to suspension by $W$. Thus, if there is an $n \geq 2$ for which $\tilde{\sigma}_{n W}^{H}$ is an isomorphism, then $\tilde{\sigma}_{W}^{H}$ must also be an isomorphism.

Since $G / H^{+} \wedge Y$ is at least as connected as $Y$, our inductive assumption gives that the map

$$
\tilde{\sigma}_{n W}^{H}(G / K): s_{*}^{n W} \pi_{V}^{G}\left(G / H^{+} \wedge Y\right)(G / K) \rightarrow \pi_{V+n W}^{G}\left(\Sigma^{n W} G / H^{+} \wedge Y\right)(G / K)
$$

is an isomorphism for every $n \geq 1$ and every proper subgroup $K$ of $G$. Thus, it suffices to show that there is an $n \geq 2$ for which $\tilde{\sigma}_{n W}^{H}(G / G)$ is an isomorphism.

Let $Z$ be the orthogonal complement of $W^{H}$ in $W$. From the cofiber sequence

$$
S Z^{+} \rightarrow D Z^{+} \simeq S^{0} \rightarrow S^{Z} \rightarrow \Sigma S Z^{+}
$$


we obtain the diagram

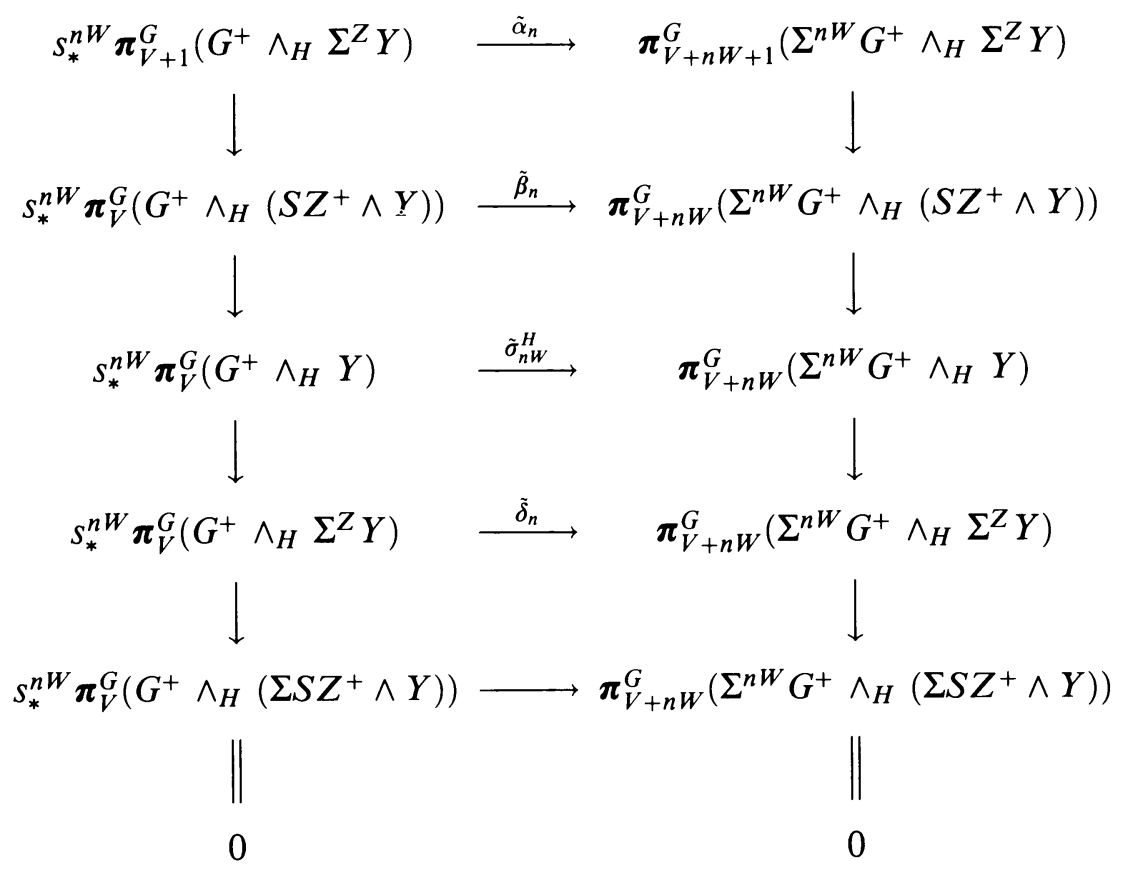

in which all the horizontal arrows are suspension maps. The bottom morphism set in each column vanishes by a simple connectivity argument. All the morphism sets in this diagram are stable with respect to suspension by the trivial representation. Our arrangement that $c^{*} Y \geq 2^{*}$ was made to ensure this stability for the top row of this diagram. The exactness of the right column follows immediately. The left column is exact at and below $s_{*}^{n W} \pi_{V}^{G}\left(G^{+} \wedge_{H} Y\right)$ by stability and the right exactness of $s_{*}^{n W}$. In order to show that the map $\tilde{\sigma}_{n W}^{H}(G / G)$ is an isomorphism, we must first show that the maps $\tilde{\beta}_{n}$ and $\tilde{\delta}_{n}$ are isomorphisms.

An induction argument on the skeleton of the $H$-CW complex $S Z$ indicates that the equivariant 1-skeleton of $S Z$ determines $\pi_{V}^{G}\left(G^{+} \wedge_{H}\left(S Z^{+} \wedge Y\right)\right)$ and $\pi_{V+n W}^{G}\left(\Sigma^{n W} G^{+} \wedge_{H}\left(S Z^{+} \wedge Y\right)\right)$. Therefore, $\tilde{\beta}_{n}$ is an isomorphism if the suspension map

$$
\tilde{\sigma}_{n W}^{J}: s_{*}^{n W} \pi_{V}^{G}\left(G^{+} \wedge_{H}\left(H / J^{+} \wedge Y\right)\right) \rightarrow \pi_{V+n W}^{G}\left(\Sigma^{n W} G^{+} \wedge_{H}\left(H / J^{+} \wedge Y\right)\right)
$$

is an isomorphism for every subgroup $J$ of $H$ which is an $H$-isotropy subgroup of $S Z$. All of these subgroups $J$ are proper subgroups of $H$. Since $G^{+} \wedge_{H}\left(H / J^{+} \wedge Y\right)$ is $G$-homeomorphic to $G / J^{+} \wedge Y$, this map $\tilde{\sigma}_{n W}^{J}$ will be an isomorphism by our second induction hypothesis if we can show that $J \in \operatorname{Iso}\left(W^{\infty}\right)$. Let $x$ be a point in $S Z$ with $H$-isotropy subgroup $J$. Let $K$ be the $G$-isotropy subgroup of $x$ regarded as a point of $W$ via the inclusions $S Z \subset Z \subset W$. Then $J=K \cap H$. Since $H \in \operatorname{Iso}\left(W^{\infty}\right)$, there is a positive integer $j$ and a point $y$ in $W^{j}$ such that $H$ is the $G$-isotropy subgroup of $y$. The $G$-isotropy subgroup of the point $(x, y)$ of $W \times W^{j}=W^{j+1}$ is $J$. Therefore, $\tilde{\beta}_{n}$ is an isomorphism.

The argument showing that $\tilde{\delta}_{n}$ is an isomorphism parallels that used to show that $\tilde{\sigma}_{W}^{H}$ is an isomorphism when $W$ is a trivial $H$-representation. Filter 
$G^{+} \wedge_{H} \Sigma^{Z} Y$ using the $H$-skeleton of $Y$ and argue that $\pi_{V}^{G}\left(G^{+} \wedge_{H} \Sigma^{Z} Y\right)$ is isomorphic to $\pi_{V}^{G}\left(G^{+} \wedge_{H} \Sigma^{Z} Y^{m+1}\right)$. Using the $H$-equivariant cofiber sequence

$$
\bigvee_{j}\left(H / J_{j}\right)^{+} \wedge S^{m} \rightarrow Y^{m} \rightarrow Y^{m+1},
$$

argue that $\tilde{\delta}_{n}$ is an isomorphism if

$$
\begin{aligned}
\tilde{\sigma}_{n W}^{J}: & S_{*}^{n W} \pi_{V}^{G}\left(G^{+} \wedge_{H} \Sigma^{Z}\left(H / J^{+} \wedge S^{m}\right)\right) \\
& \rightarrow \pi_{V+n W}^{G}\left(\Sigma^{n W} G^{+} \wedge_{H} \Sigma^{Z}\left(H / J^{+} \wedge S^{m}\right)\right)
\end{aligned}
$$

is an isomorphism for every subgroup $J$ of $H$. The spaces $S^{Z+m}$ and $S^{V}$ are $H$-homeomorphic. Thus, there are $G$-homeomorphisms

$$
G^{+} \wedge_{H} \Sigma^{Z}\left(H / J^{+} \wedge S^{m}\right) \cong G^{+} \wedge_{J} S^{Z+m} \cong G^{+} \wedge_{J} S^{V} \cong G / J^{+} \wedge S^{V},
$$

which allow us to replace $G^{+} \wedge_{H} \Sigma^{Z}\left(H / J^{+} \wedge S^{m}\right)$ by $G / J^{+} \wedge S^{V}$. The map

$$
\tilde{\sigma}_{n W}^{J}: s_{*}^{n W} \pi_{V}^{G}\left(G / J^{+} \wedge S^{V}\right) \rightarrow \pi_{V+n W}^{G}\left(\Sigma^{n W} G / J^{+} \wedge S^{V}\right)
$$

is an isomorphism by Lemma 4.5(ii). Therefore, $\tilde{\delta}_{n}$ is an isomorphism.

If $\tilde{\alpha}_{n}(G / G)$ were an epimorphism for some $n$, then a simple chase of the diagram above would imply that $\tilde{\sigma}_{n W}^{H}(G / G)$ was an isomorphism for that $n$, completing the proof. Note that, by Lemma $4.5(\mathrm{iv}), \tilde{\alpha}_{n}(G / G)$ is an epimorphism if the suspension homomorphism

$$
\begin{aligned}
\alpha_{n}(G / G): & \pi_{V+1}^{G}\left(G^{+} \wedge_{H} \Sigma^{Z} Y\right)(G / G) \\
& \rightarrow \pi_{V+n W+1}^{G}\left(\Sigma^{n W} G^{+} \wedge_{H} \Sigma^{Z} Y\right)(G / G)
\end{aligned}
$$

is an epimorphism. If $H=G$, then $W=Z$ and Namboodiri's theorem gives that $\alpha_{n}(G / G)$ is an epimorphism. Therefore, we assume that $H \neq G$.

Recall that $L$ is the tangent space of $G / H$ at $e H$. We must consider two cases depending on whether or not $L^{H}$ is zero. Assume first that $L^{H} \neq 0$. The orbit $G / H$ embeds as a $G$-space into $n W$ for some $n \geq 1$. Also, the groups

$$
\begin{gathered}
\pi_{V+n W}^{G}\left(\Sigma^{n W} G^{+} \wedge_{H}\left(S Z^{+} \wedge Y\right)\right), \quad \pi_{V+n W}^{G}\left(\Sigma^{n W} G^{+} \wedge_{H} Y\right), \\
\pi_{V+n W}^{G}\left(\Sigma^{n W} G^{+} \wedge_{H} \Sigma^{Z} Y\right)
\end{gathered}
$$

are all stable with respect to suspension by both $W$ and the trivial representation. Thus, we may apply Wirthmüller's isomorphism [15, Corollary II.6.5] to these groups and obtain that, for all $n \geq 1$, there are isomorphisms

$$
\begin{aligned}
\pi_{V+n W}^{G}\left(\Sigma^{n W} G^{+} \wedge_{H}\left(S Z^{+} \wedge Y\right)\right)(G / G) & \cong \pi_{V+n W}^{G}\left(\Sigma^{n W} G^{+} \wedge_{H}\left(S Z^{+} \wedge Y\right)\right) \\
& \cong \pi_{V+n W}^{H}\left(\Sigma^{n W+L} S Z^{+} \wedge Y\right), \\
\pi_{V+n W}^{G}\left(\Sigma^{n W} G^{+} \wedge_{H} Y\right)(G / G) & \cong \pi_{V+n W}^{G}\left(\Sigma^{n W} G^{+} \wedge_{H} Y\right) \\
& \cong \pi_{V+n W}^{H}\left(\Sigma^{n W+L} Y\right) \\
\pi_{V+n W}^{G}\left(\Sigma^{n W} G^{+} \wedge_{H} \Sigma^{Z} Y\right)(G / G) & \cong \pi_{V+n W}^{G}\left(\Sigma^{n W} G^{+} \wedge_{H} \Sigma^{Z} Y\right) \\
& \cong \pi_{V+n W}^{H}\left(\Sigma^{n W+L+Z} Y\right) .
\end{aligned}
$$

By easy connectivity arguments, the groups on the right-hand sides of each of these equations vanish. Since the maps $\tilde{\beta}_{n}$ and $\tilde{\delta}_{n}$ are isomorphisms in the diagram above, the groups

$$
s_{*}^{W} \pi_{V}^{G}\left(G^{+} \wedge_{H}\left(S Z^{+} \wedge Y\right)\right)(G / G) \text { and } s_{*}^{W} \pi_{V}^{G}\left(G^{+} \wedge_{H} \Sigma^{Z} Y\right)(G / G)
$$


also vanish. Therefore, $s_{*}^{W} \pi_{V}^{G}\left(G^{+} \wedge_{H} Y\right)(G / G)$ vanishes, $\tilde{\sigma}_{n W}^{H}(G / G)$ is an isomorphism, and we do not need to prove that $\alpha_{n}(G / G)$ is an epimorphism.

Now assume that $L^{H}=0$. Let $p=\left|W^{H}\right|$. Since the groups

$$
\pi_{V+1}^{G}\left(G^{+} \wedge_{H} \Sigma^{Z} Y\right)(G / G) \text { and } \pi_{V+n W+1}^{G}\left(\Sigma^{n W} G^{+} \wedge_{H} \Sigma^{Z} Y\right)(G / G)
$$

are stable with respect to suspension by the trivial representation, we can suspend both $p$-times and use the $G$-homeomorphisms

$$
\Sigma^{p} G^{+} \wedge_{H} \Sigma^{Z} Y \cong G^{+} \wedge_{H} \Sigma^{Z+p} Y \cong G^{+} \wedge_{H} \Sigma^{W} Y \cong \Sigma^{W}\left(G / H^{+} \wedge Y\right)
$$

to convert the problem of showing that $\alpha_{n}(G / G)$ is an epimorphism to that of showing that the suspension homomorphism

$$
\begin{aligned}
\sigma_{n}(G / G): & \pi_{V+p+1}^{G}\left(\Sigma^{W}\left(G / H^{+} \wedge Y\right)\right)(G / G) \\
& \rightarrow s^{*} \pi_{V+n W+p+1}^{G}\left(\Sigma^{(n+1) W}\left(G / H^{*} \wedge Y\right)\right)(G / G)
\end{aligned}
$$

is an epimorphism. By Namboodiri's Theorem,

$$
\begin{aligned}
\sigma_{n}(G / H): & \pi_{V+p+1}^{G}\left(\Sigma^{W}\left(G / H^{+} \wedge Y\right)\right)(G / H) \\
& \rightarrow s^{*} \pi_{V+n W+p+1}^{G}\left(\Sigma^{(n+1) W}\left(G / H^{+} \wedge Y\right)\right)(G / H)
\end{aligned}
$$

is an epimorphism. We want to use transfer maps to exploit the fact that $\sigma_{n}(G / H)$ is an epimorphism. Select $n \geq 2$ such that $G / H$ embeds as a $G$ space in $n W$. Then, by Definition 4.2(ii), there is an unstable transfer map

$$
\tau_{2}: S^{V+n W+p+1} \rightarrow \Sigma^{V+n W+p+1} G / H^{+}
$$

in $\mathscr{B}_{G}(V+n W+p+1)$. The suspension factor

$$
s: \mathscr{B}_{G}(V+p+1) \rightarrow \mathscr{B}_{G}(V+n W+p+1)
$$

is an epimorphism on morphism sets, so we can pick a map $\tau_{1}$ in $\mathscr{B}_{G}(V+p+1)$ which suspends to $\tau_{2}$. Since $\tau_{1}$ suspends to $\tau_{2}$, the diagram

$$
\begin{gathered}
\pi_{V+p+1}^{G}\left(\Sigma^{W}\left(G / H^{+} \wedge Y\right)\right)(G / H) \stackrel{\sigma_{n}(G / H)}{\longrightarrow} \pi_{V+n W+p+1}^{G}\left(\Sigma^{(n+1) W}\left(G / H^{+} \wedge Y\right)\right)(G / H) \\
\tau_{1} \downarrow \\
\pi_{V+p+1}^{G}\left(\Sigma^{W}\left(G / H^{+} \wedge Y\right)\right)(G / G) \stackrel{\sigma_{n}(G / G)}{\longrightarrow} \pi_{V+n W+p+1}^{G}\left(\Sigma^{(n+1) W}\left(G / H^{+} \wedge Y\right)\right)(G / G)
\end{gathered}
$$

commutes. From the diagram, it follows that $\sigma_{n}(G / G)$ must be an epimorphism if $\tau_{2}$ is. If $G$ were a finite group and $\pi_{V+n W+p+1}^{G}\left(\Sigma^{(n+1) W}\left(G / H^{+} \wedge Y\right)\right)$ were a stable morphism set, then Wirthmüller's isomorphism [15] would imply that

$$
\boldsymbol{\pi}_{V+n W+p+1}^{G}\left(\Sigma^{(n+1) W}\left(G / H^{+} \wedge Y\right)\right)
$$

was a $G / H$-projective Mackey functor $[5,11]$. This would force $\tau_{2}$ to be an epimorphism. The remainder of the proof of the theorem consists of the introduction of a trick for bypassing the problems that $G$ may not be finite and that the morphism sets are unstable. Since we have chosen $n \geq 2$, Namboodiri's theorem indicates that $\pi_{V+n W+p+1}^{G}\left(\Sigma^{(n+1) W}\left(G / H^{+} \wedge Y\right)\right)$ is stable with respect to suspension by $W$. Thus, Wirthmüller's isomorphism may be applied to obtain isomorphisms

$$
\pi_{V+n W+p+1}^{G}\left(\Sigma^{(n+1) W}\left(G / H^{+} \wedge Y\right)\right)(G / G) \cong\left[S^{V+n W+p+1}, \Sigma^{(n+1) W+L} Y\right]_{H}
$$


and

$$
\begin{aligned}
& \pi_{V+n W+p+1}^{G}\left(\Sigma^{(n+1) W}\left(G / H^{+} \wedge Y\right)\right)(G / H) \\
& \cong\left[\Sigma^{V+n W+p+1} G / H^{+}, \Sigma^{(n+1) W+L} Y\right]_{H} .
\end{aligned}
$$

Recall from Definition 4.2(ii) that the unstable transfer $\tau_{2}$ is the suspension of the composite

$$
\tau: S^{n W} \stackrel{f}{\longrightarrow} G^{+} \wedge_{H} S^{N} \stackrel{e}{\longrightarrow} G^{+} \wedge_{H} S^{n W} \cong \Sigma^{n W} G / H,
$$

where the $H$-representation $N$ is the orthogonal complement of $L$ in $n W$. The first map $f$ in this composite is obtained by collapsing out a $G$-equivariant tubular neighborhood of $G / H$ in $n W$. The second map $e$ is derived from the inclusion of $N$ into $n W$. Under the identifications obtained from Wirthmüller's isomorphism, $\tau_{2}$ becomes the map

$$
\left[\Sigma^{V+n W+p+1} G / H^{+}, \Sigma^{(n+1) W+L} Y\right]_{H} \stackrel{\left(\Sigma^{V+p+1} \tau\right)^{*}}{\longrightarrow}\left[S^{V+n W+p+1}, \Sigma^{(n+1) W+L} Y\right]_{H} \text {. }
$$

Thus, to show that $\tau_{2}$ is an epimorphism, it suffices to show that

$$
\begin{aligned}
\left(\Sigma^{V+p+1} f\right)^{*}: & {\left[\Sigma^{V+p+1} G^{+} \wedge_{H} S^{N}, \Sigma^{(n+1) W+L} Y\right]_{H} } \\
& \rightarrow\left[S^{V+n W+p+1}, \Sigma^{(n+1) W+L} Y\right]_{H}
\end{aligned}
$$

and

$$
\begin{aligned}
\left(\Sigma^{V+p+1} e\right)^{*}: & {\left[\Sigma^{V+n W+p+1} G / H^{+}, \Sigma^{(n+1) W+L} Y\right]_{H} } \\
& \rightarrow\left[\Sigma^{V+p+1} G^{+} \wedge_{H} S^{N}, \Sigma^{(n+1) W+L} Y\right]_{H}
\end{aligned}
$$

are epimorphisms.

To see that $\left(\Sigma^{V+p+1} f\right)^{*}$ is an epimorphism, recall from Construction II.5.5 and Lemma II.5.9 of [15] that collapsing out an equivariant neighborhood of $\{e\}^{+} \wedge_{H} S^{N}$ in $G^{+} \wedge_{H} S^{N}$ gives an $H$-map

$$
u: G^{+} \wedge_{H} S^{N} \rightarrow S^{n W}
$$

such that the composite $u f$ is $H$-homotopic to the identity map. It follows that $\left(\Sigma^{V+p+1} f\right)^{*}$ is a split epimorphism.

The map $\left(\Sigma^{V+p+1} e\right)^{*}$ is derived from the second map in the cofiber sequence

$$
S L^{+} \rightarrow D L^{+} \simeq S^{0} \rightarrow S^{L} .
$$

Thus, to show that $\left(\Sigma^{V+p+1} e\right)^{*}$ is an epimorphism, it suffices to show that the group

$$
\left[\Sigma^{V+p+1} G^{+} \wedge_{H} \Sigma^{N} S L^{+}, \Sigma^{(n+1) W+L} Y\right]_{H}
$$

is zero. The group

$$
\left[\Sigma^{V+p+1} G^{+} \wedge_{H} \Sigma^{N} S L^{+}, \Sigma^{(n+1) W}\left(G / H^{+} \wedge Y\right)\right]_{G}
$$

is stable with respect to suspension by both $W$ and the trivial representation. Thus, Wirthmüller's result gives the first of the following two isomorphisms:

$$
\begin{aligned}
{\left[\Sigma^{V+p+1} G^{+} \wedge_{H} \Sigma^{N}\right.} & \left.S L^{+}, \Sigma^{(n+1) W+L} Y\right]_{H} \\
& \cong\left[\Sigma^{V+p+1} G^{+} \wedge_{H} \Sigma^{N} S L^{+}, \Sigma^{(n+1) W}\left(G / H^{+} \wedge Y\right)\right]_{G} \\
& \cong\left[\Sigma^{V+p+1+N} S L^{+}, \Sigma^{(n+1) W}\left(G / H^{+} \wedge Y\right)\right]_{H} .
\end{aligned}
$$


The second isomorphism holds since $\Sigma^{V+p+1} G^{+} \wedge_{H} \Sigma^{N} S L^{+}$is the free $G$-space generated by the $H$-space $\Sigma^{V+p+1+N} S L^{+}$. By an elementary connectivity argument,

$$
\left[\Sigma^{V+p+1+N} S L^{+}, \Sigma^{(n+1) W}\left(G / H^{+} \wedge Y\right)\right]_{H}
$$

is zero. It follows that $\left(\Sigma^{V+p+1} e\right)^{*}$, and therefore $\tau_{2}$ and $\tilde{\alpha}_{n}(G / G)$, are epimorphisms.

\section{CHANGE OF UNIVERSE AND HOMOTOPY GROUPS}

The passage from stable homotopy groups computed with respect to the $G$ universe $V^{\infty}$ to those computed with respect to a complete $G$-universe $U$ is made using the change of universe functors of [15, §II.1]. Recall that if $i: U^{\prime} \rightarrow U$ is the inclusion of a $G$-subuniverse, then there is a change of universe functor $i_{*}$ taking spectra indexed on $U^{\prime}$ to spectra indexed on $U$. Let $s^{*}$ be the forgetful functor that converts $U$-Mackey functors to $U^{\prime}$-Mackey functors. Then for any $G$-spectrum $D$ indexed on $U^{\prime}$, the functor $i_{*}$ induces a natural transformation of $U^{\prime}$-Mackey functors

$$
\sigma: \boldsymbol{\pi}_{*}^{G}(D) \rightarrow s^{*} \boldsymbol{\pi}_{*}^{G}\left(i_{*} D\right)
$$

which we think of as a stabilization of the $U^{\prime}$-stable homotopy groups of $D$. The following proposition gives conditions under which the adjoint of this map is an isomorphism.

Proposition 6.1. Let $i: U^{\prime} \rightarrow U$ be the inclusion of a $G$-subuniverse and $D$ be a $(-1)$-connected $G-C W$ spectrum indexed on $U^{\prime}$. Then $i_{*} D$ is a (-1)-connected $G-C W$ spectrum indexed on $U$ and the stabilization map

$$
\tilde{\sigma}: s_{*} \pi_{0}^{G}(D) \rightarrow \pi_{0}^{G}\left(i_{*} D\right)
$$

is an isomorphism.

Proof. That $i_{*} D$ is a $G$-CW spectrum follows from the colimit-preserving properties of $i_{*}$ [15, Proposition II.1.4]. Moreover, $i_{*}$ takes the skeletal filtration of $D$ to that of $i_{*} D$. We may assume that the $(-1)$-skeleton of $D$ is the point spectrum and the 0 -skeleton $D^{0}$ is a wedge of $U^{\prime}$-suspension spectra of orbits. The 1-skeleton of $D$ is then described by a cofiber sequence

$$
\bigvee_{j} \Sigma^{\infty} G / K_{j}^{+} \rightarrow D^{0} \rightarrow D^{1} .
$$

The 1-skeleton of $i_{*} D$ is described by the cofiber sequence obtained by applying $i_{*}$ to this sequence. Thus, the sequences

$$
\boldsymbol{\pi}_{0}^{G}\left(\bigvee_{j} \Sigma^{\infty} G / K_{j}^{+}\right) \rightarrow \pi_{0}^{G}\left(D^{0}\right) \rightarrow \boldsymbol{\pi}_{0}^{G}\left(D^{1}\right) \rightarrow 0
$$

and

$$
\boldsymbol{\pi}_{0}^{G}\left(\bigvee_{j} \Sigma^{\infty} G / K_{j}^{+}\right) \rightarrow \pi_{0}^{G}\left(i_{*} D^{0}\right) \rightarrow \pi_{0}^{G}\left(i_{*} D^{1}\right) \rightarrow 0,
$$

in which the zeros on the right come from Proposition 3.2, display $\pi_{0}^{G}\left(D^{1}\right)$ and $\pi_{0}^{G}\left(i_{*} D^{1}\right)$ as cokernels. The first two terms in each of these exact sequences are direct sums of representable functors. Therefore, by Lemma 4.5(ii), the stabilization maps

$$
\tilde{\sigma}: s_{*} \pi_{0}^{G}\left(\bigvee_{j} \Sigma^{\infty} G / K_{j}^{+}\right) \rightarrow \pi_{0}^{G}\left(\bigvee_{j} \Sigma^{\infty} G / K_{j}^{+}\right)
$$


and

$$
\tilde{\sigma}: s_{*} \boldsymbol{\pi}_{0}^{G}\left(D^{0}\right) \rightarrow \boldsymbol{\pi}_{0}^{G}\left(i_{*} D^{0}\right)
$$

are isomorphisms. Since $s_{*}$ preserves cokernels and $\tilde{\sigma}$ is a map of sequences, the stabilization map

$$
\tilde{\sigma}: s_{*} \pi_{0}^{G}\left(D^{1}\right) \rightarrow \pi_{0}^{G}\left(i_{*} D^{1}\right)
$$

is an isomorphism. The conclusion of the lemma follows because the inclusions $D^{1} \subset D$ and $i_{*} D^{1} \subset i_{*} D$ induce isomorphisms on $\pi_{0}^{G}$.

Propositions 3.6(ii) and 6.1 and Theorems 2.1 and 5.1 combine to give a preliminary version of the second form of the unstable isomorphism theorem.

Proposition 6.2. Let $Y$ be a based $G$-CW complex and $V$ be a G-representation with $\left|V^{G}\right| \geq 2$. If $Y$ is $(V-1)$-connected, then

$$
\tilde{h}: s_{*} \boldsymbol{\pi}_{V}^{G}(Y) \rightarrow \mathbf{H}_{V}^{G}(Y)
$$

is an isomorphism.

Proof. By Theorem 5.1 and Proposition 3.6(ii), the stabilization map

$$
\tilde{\sigma}_{1}:\left(s_{1}\right)_{*} \pi_{V}^{G}(Y) \rightarrow \pi_{V}^{G}\left(\Sigma^{\infty} Y\right)
$$

is an isomorphism if the stable group is computed with respect to the universe $V^{\infty}$ and $\left(s_{1}\right)_{*}$ is the functor associated to the inclusion of $V$ into $V^{\infty}$ as the first summand. Taking $D=\Sigma^{-V} \Sigma^{\infty} Y$ in Proposition 6.1, we obtain that the map

$$
\tilde{\sigma}_{2}:\left(s_{2}\right)_{*} \boldsymbol{\pi}_{V}^{G}\left(\Sigma^{\infty} Y\right) \rightarrow \boldsymbol{\pi}_{V}^{G}\left(\Sigma^{\infty} Y\right)
$$

is an isomorphism if the group on the left is computed with respect to the $G$ universe $V^{\infty}$, the group on the right is computed with respect to a complete $G$ universe $U$, and the functor $\left(s_{2}\right)_{*}$ is that associated to an inclusion $V^{\infty} \subset U$. By Theorem 2.1, for each subgroup $K$ of $G$, the map

$$
h: \pi_{V}^{K}\left(\Sigma^{\infty} Y\right) \rightarrow H_{V}^{K}(Y)
$$

is an isomorphism if the group $\pi_{V}^{K}\left(\Sigma^{\infty} Y\right)$ is computed with respect to a complete $G$-universe $U$. Thus $h$, regarded as a natural transformation between Mackey functors, is an isomorphism:

$$
h: \boldsymbol{\pi}_{V}^{G}\left(\Sigma^{\infty} Y\right) \rightarrow \mathbf{H}_{V}^{G}(Y) .
$$

Combining these isomorphisms, we obtain the unstable Hurewicz isomorphism

$$
\tilde{h}: s_{*} \boldsymbol{\pi}_{V}^{G}(Y) \cong\left(s_{2}\right)_{*}\left(s_{1}\right)_{*} \boldsymbol{\pi}_{V}^{G}(Y) \rightarrow \mathbf{H}_{V}^{G}(Y) .
$$

Here $s_{*}$ is the functor derived from an inclusion of $V$ in a complete universe $U$ and Lemma $4.5(\mathrm{v})$ is used to identify $s_{*} \pi_{V}^{G}(Y)$ and $\left(s_{2}\right)_{*}\left(s_{1}\right)_{*} \pi_{V}^{G}(Y)$.

\section{7. $V$ - AND $\left|V^{*}\right|$-CONNECTIVITY}

In this section, we prove our extension of Waner's result on the relation between $V$-and $\left|V^{*}\right|$-connectivity. The absolute version of the second form of the unstable Hurewicz theorem (Theorem 1.7) is then proved using this relation.

Proof of Lemma 1.2. Let $K$ be a subgroup of $G$. If $K=\{e\}$, then the $K-V$ and $K-\left|V^{*}\right|$-conditions in all three parts of the lemma are equivalent. Thus, 
the lemma may be proved inductively using the descending chain condition on the subgroups $K$ of $G$. Our inductive assumption for each of the three parts of the lemma will be that, for each proper subgroup $J$ of $K$, the $J-V$ - and $J-\left|V^{*}\right|$-conditions are equivalent. Let $W$ be the orthogonal complement of $V^{K}$ in $V$ and consider the cofiber sequence

$$
S W^{+} \rightarrow S^{0} \rightarrow S^{W} \rightarrow \Sigma S W^{+} \rightarrow S^{1}
$$

For part (i), let $m$ be an integer with $0 \leq m \leq\left|V^{K}\right|$. Assume first that $Y$ is $G-\left|V^{*}\right|$-connected. From the middle three terms of our cofiber sequence, we obtain the exact sequence

$$
\left[\Sigma^{m+1} S W^{+}, Y\right]_{K} \rightarrow \pi_{W+m}^{K}(Y) \rightarrow \pi_{m}^{K}(Y)
$$

of homotopy sets. The right-most term in this sequence vanishes since $Y^{K}$ is $\left|V^{K}\right|$-connected by assumption. The left-most term in this sequence vanishes because $\Sigma^{m+1} S W^{+}$is a $K$-CW complex whose equivariant cells have the form $K / J^{+} \wedge e^{n}$, where $J$ is a proper subgroup of $K$ and $n \leq\left|W^{J}\right|+m \leq\left|V^{J}\right|$. Thus, $\pi_{W+m}^{K}(Y)$ vanishes as required.

Now assume that $Y$ is $V$-connected. The three left-most terms of our cofiber sequence yield the exact sequence

$$
\pi_{W+m}^{K}(Y) \rightarrow \pi_{m}^{K}(Y) \rightarrow\left[\Sigma^{m} S W^{+}, Y\right]_{K}
$$

of homotopy sets. The left-most term in this sequence vanishes by our assumption that $Y$ is $V$-connected. The right-most term vanishes because, by induction, $Y$ is $J-\left|V^{*}\right|$-connected for every proper subgroup $J$ of $K$. Thus, $\pi_{m}^{K}(Y)$ vanishes as required.

Part (ii) is a special case of part (iii). For part (iii), let $f: Y \rightarrow Z$ be a $G$-map and $m$ be a nonnegative integer.

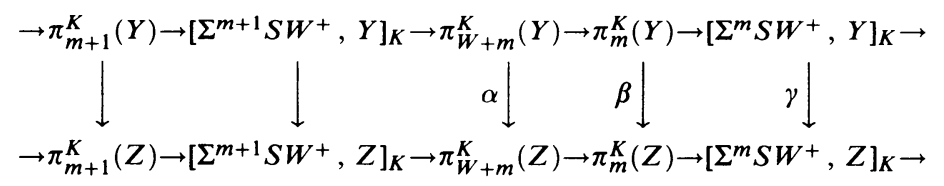

obtained by applying the functors $[?, Y]_{K}$ and $[?, Z]_{K}$ to our cofiber sequence. The vertical arrows are all induced by $f$. By our inductive hypothesis, $f$ is $J-\left|V^{*}\right|$-connected for every proper subgroup $J$ of $K$. It follows by an induction on the $K$-skeleton of $S W$ that the map $\gamma$ is an injection for $0 \leq m \leq\left|V^{K}\right|$ and a surjection for $0 \leq m \leq\left|V^{K}\right|+1$. This observation and standard five lemma arguments suffice to show that $\alpha$ is an isomorphism/epimorphism for the appropriate values of $m$ if and only if $\beta$ is. Note that, since a $G-V$. equivalence is required to be a $G-0^{*}$-equivalence, we need not show that $\alpha$ being an epimorphism for $m=0$ implies that $\beta$ is also. Of course, when $m=1$, the usual precautions must be taken since the groups may be nonabelian. Also, when $m=0$, the action of the groups $\left[\Sigma^{m+1} S W^{+}, Y\right]_{K}$ and $\left[\Sigma^{m+1} S W^{+}, Z\right]_{K}$ on the sets $\pi_{W+m}^{K}(Y)$ and $\pi_{W+m}^{K}(Z)$ must be used to make up for the lack of a multiplication on $\pi_{W+m}^{K}(Y)$ and $\pi_{W+m}^{K}(Z)$.

Remarks 7.1. The proof of the equivalence of the $V$ - and $\left|V^{*}\right|$-connectivity of a $G$-space $Y$ can easily be altered to show that $Y$ is homologically $V$ connected if and only if it is homologically $\left|V^{*}\right|$-connected. The equivalence of 
homological $V$-connectedness and homological $\left|V^{*}\right|$-connectedness for a pair $(Y, B)$ follows from the equivalence of the two conditions for spaces applied to the cofiber of the inclusion of $B$ into $Y$.

We can now prove the absolute version of our second type of an unstable Hurewicz Isomorphism Theorem.

Proof of Theorem 1.7. Throughout this proof, we freely use Lemma 1.2 and Remarks 7.1 to equate $(V-1)$ - and $\left|(V-1)^{*}\right|$-connectivity in both homotopy and homology. To see that condition (a) implies condition (b), note that, if $Y$ is $G-\left|(V-1)^{*}\right|$-connected, then it must be $G-1^{*}$-connected. Also, by Proposition 2.4(i), it must be homologically $(V-1)$-connected. To show that (b) implies (a), it suffices to show that, if $Y$ is simply $G$-connected and homologically $G-\left|(V-1)^{*}\right|$-connected, then it is $G-\left|(V-1)^{*}\right|-$ connected. That is, we must show that $\pi_{m}^{K}(Y)=0$ for every subgroup $K$ of $G$ and every integer $m$ with $0 \leq m \leq\left|(V-1)^{K}\right|$. The cases $m=0$ and $m=1$ follow from the assumption of simple $G$-connectivity. The remaining cases may be handled by an induction over $K$ and $m$. In this induction, Propositions 1.9 and 6.2 allow us to derive the vanishing of $\pi_{m}^{K}(Y)$ from the assumed vanishing of $\mathbf{H}_{m}^{K}(Y)$. Proposition 6.2 and Lemma 1.2 indicate that condition (a) implies that $\tilde{h}$ is an isomorphism. The vanishing of the homology groups in the required dimensions follows from Proposition 2.4(i). The vanishing of the homotopy groups in the required dimensions follows from Lemma 1.2.

\section{The Relative Hurewicz Theorem and the Whitehead theorem}

Here we prove both the relative Hurewicz Isomorphism Theorem (Theorem 1.8) and the Whitehead theorem (Theorem 1.11). The following relative version of Proposition 6.2 is the key to our proof of the relative Hurewicz theorem.

Proposition 8.1. Let $(Y, B)$ be a based $G$-CW pair with $Y$ and $B$ both simply $G$-connected and $V$ be a G-representation with $\left|V^{G}\right| \geq 2$. If $(Y, B)$ is $(V-1)$ connected, then $\pi_{V}^{G}(Y, B)$ is a $V$-Mackey functor and

$$
\tilde{h}: s_{*} \boldsymbol{\pi}_{V}^{G}(Y, B) \rightarrow \mathbf{H}_{V}^{G}(Y, B)
$$

is an isomorphism, where $s_{*}$ is the functor derived from the inclusion of $V$ into a complete $G$-universe $U$.

Proof. By attaching $G$-cells to $B$, one may form a $G$-pair $(Z, C)$ which contains $(Y, B)$ as a subpair and such that $Z$ and $C$ are $\left|(V-1)^{*}\right|$-connected. The pair $(Z, C)$ must be $\left|(V-1)^{*}\right|$-connected since both $Z$ and $C$ are. Let $C i$ and $C j$ be the homotopy cofibers of the inclusions $i: B \rightarrow Y$ and $j: C \rightarrow Z$. Since all the $G$-cells were added to $B$, the inclusion of $C i$ in $C j$ is a homotopy equivalence. By the equivariant Homotopy Excision Theorem [7, Satz (2.2)], the natural maps

$$
\pi_{V}^{K}(Y, B) \rightarrow \pi_{V}^{K}(C i), \quad \pi_{V}^{K}(Z, C) \rightarrow \pi_{V}^{K}(C j)
$$

are isomorphisms for every subgroup $K$ of $G$. Thus, the map

$$
\pi_{V}^{K}(Y, B) \rightarrow \pi_{V}^{K}(Z, C)
$$


is an isomorphism for every subgroup $K$. As noted in Examples 1.6(ii), $\pi_{V}^{G}(Z, C)$ is a $V$-Mackey functor instead of just a $(V-1)$-Mackey functor. Therefore, so is $\pi_{V}^{G}(Y, B)$. To complete the proof, it suffices to show that the map

$$
\tilde{h}: s_{*} \boldsymbol{\pi}_{V}^{G}(Z, C) \rightarrow \mathbf{H}_{V}^{G}(Z, C)
$$

is an isomorphism. This follows from the absolute Hurewicz Isomorphism Theorem (Theorem 1.7) and the following commuting diagram whose rows are exact:

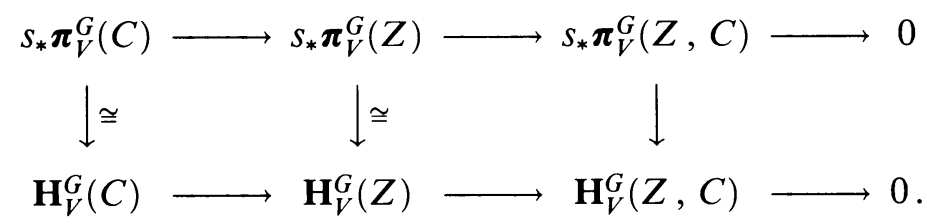

Proof of Theorem 1.8. Let $C i$ be the homotopy cofiber of the inclusion

$$
i: B \rightarrow Y .
$$

Note that $C i$ is simply $G$-connected since $Y$ and $B$ are. Assume that $(Y, B)$ is $\left|(V-1)^{*}\right|$-connected. Then by the equivariant Homotopy Excision Theorem [7, Satz (2.2)], $C i$ is $\left|(V-1)^{*}\right|$-connected. It follows by Proposition 2.4(i) and Remarks 7.1 that $(Y, B)$ is homologically $G-(V-1)$-connected. Thus, condition (a) implies condition (b). The proof of the remainder of the theorem parallels the analogous part of the proof of Theorem 1.7. The only required changes are that Proposition 8.1 is used in the place of Proposition 6.2 and that, when Proposition 2.4(i) is used, it is applied to $C i$ instead of $Y$.

In order to obtain a Whitehead theorem applicable to $G$-spaces that are not simply $G$-connected, we adopt a method of proof more complicated than simply deriving the Whitehead result from our Hurewicz theorems. The proof of the following lemma is an obvious extension of the proof that any $G$-space is weakly $G$-equivalent to a $G$-CW complex.

Lemma 8.2. Let $f: Y \rightarrow Z$ be a G-map between $G$-CW complexes with $c^{G} f \geq 0$. Then there exists G-CW complexes $Y^{\prime}$ and $Z^{\prime}$ and G-maps $f^{\prime}: Y^{\prime} \rightarrow$ $Z^{\prime}, g: Y^{\prime} \rightarrow Y$, and $h: Z^{\prime} \rightarrow Z$ such that the following hold:

(i) $g$ and $h$ are G-homotopy equivalences,

(ii) $f^{\prime}$ is cellular and restricts to a homeomorphism from the G-n-skeleton of $Y^{\prime}$ to that of $Z^{\prime}$ for all $n \leq c^{G} f$, and

(iii) the diagram

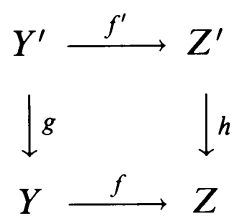

commutes up to G-homotopy.

Proof of Theorem 1.11. If $f: Y \rightarrow Z$ is a $G-V$-equivalence and $W$ is a $G$ representation with $\left|W^{*}\right| \leq\left|V^{*}\right|$, then $f$ is a $G$-W -equivalence by Lemma 1.2. 
To show that $f$ is also a homology $G$ - $V$-equivalence, it suffices, by Remarks 7.1 , to show that, for each subgroup $K$ of $G$, the map

$$
f_{*}: H_{n}^{K}(Y) \rightarrow H_{n}^{K}(Z)
$$

is an isomorphism if $0 \leq n<\left|V^{K}\right|$ and an epimorphism if $n=\left|V^{K}\right|$. For each subgroup $K$, by applying Lemma 8.2 to $f$, regarded as a $K$-map, we may assume that $f$ is cellular with respect to the $K$-skeleton of $Y$ and $Z$ and that it restricts to a $K$-homeomorphism from the $K$-n-skeleton of $Y$ to the $K$-n-skeleton of $Z$ for all $n \leq\left|V^{K}\right|$. Since $H_{n}^{G}(Y)$ is determined by the $K-(n+1)$-skeleton of $Y$, it follows immediately that $f_{*}$ is an isomorphism for $0 \leq n<\left|V^{K}\right|$. Moreover, since the inclusion of the $K-n$-skeleton $Y^{n}$ of $Y$ induces an epimorphism

$$
f_{*}: H_{n}^{K}\left(Y^{n}\right) \rightarrow H_{n}^{K}(Y),
$$

it follows that $f_{*}$ is an epimorphism for $n=\left|V^{K}\right|$.

If $Y$ and $Z$ are simply $G$-connected and $f: Y \rightarrow Z$ is a homology $G-V$ equivalence, then to show that $f$ is also a $G$ - $V$-equivalence, it suffices to show that, for each subgroup $K$ of $G$, the map

$$
f_{*}: \pi_{n}^{K}(Y) \rightarrow \pi_{n}^{K}(Z)
$$

is an isomorphism if $0 \leq n<\left|V^{K}\right|$ and an epimorphism if $n=\left|V^{K}\right|$. The cases $n=0$ and $n=1$ are immediate because of our assumption of simple $G$ connectivity. Thus we have nothing to prove for a particular subgroup $K$ unless $\left|V^{K}\right| \geq 2$. For those subgroups $K$ of $G$ with $\left|V^{K}\right| \geq 2$, we may convert $f$ into an inclusion with the usual mapping cylinder construction. The relative Hurewicz theorem (Theorem 1.8) and Proposition 1.9 then indicate that the appropriate relative homotopy groups vanish.

\section{ACKNOWLEDGMENTS}

I would like to thank Stefan Waner for sharing the unpublished manuscript of [21]. I would also like to thank Doug Anderson and Jack Ucci for helpful conversations and for reminding me of Whitehead's result [23]. Finally, I would like to thank both the Alexander von Humboldt Foundation and Sonderforschungsbereich 170 in Göttingen for their support and hospitality during the completion of this paper.

\section{REFERENCES}

1. G. E. Bredon, Equivariant cohomology theories, Lecture Notes in Math., vol. 34, Springer, 1967.

2. __ Introduction to compact transformation groups, Academic Press, 1972.

3. J. Caruso and S. Waner, An approximation theorem for equivariant loop spaces in the compact Lie case, Pacific J. Math. 117 (1985), 27-49.

4. T. tom Dieck and T. Petrie, Geometric modules over the Burnside ring, Invent. Math. 47 (1978), 273-287.

5. A. Dress, Contributions to the theory of induced representations, Lecture Notes in Math., vol. 342, Springer, 1973, pp. 183-240.

6. A. Grothendieck, Sur quelques points d'algèbre homologique, Tôhoku Math. J. 9 (1957), 119-221. 
7. H. Hauschild, Äquivariante Homotopie I, Arch. Math. 29 (1977), 158-165.

8. ___ Zerspaltung äquivarianter Homotopiemengen, Math. Ann. 230 (1977), 279-292.

9. S. Illman, Equivariant singular homology and cohomology for actions of compact Lie groups, Lecture Notes in Math., vol. 298, Springer, 1972, pp. 403-415.

10. __ Equivariant singular homology and cohomology I, Mem. Amer. Math. Soc., No. 156 (1975).

11. L. G. Lewis, Jr., An introduction to Mackey functors (in preparation).

12. __ The ordinary $\mathrm{RO}(G)$-graded cohomology of a point for cyclic p-groups (in preparation).

13. The $\mathrm{RO}(G)$-graded equivariant ordinary cohomology of complex projective spaces with linear $\mathbb{Z} / p$ actions, Lecture Notes in Math., vol. 1361, Springer, 1988, pp. 53-122.

14. L. G. Lewis, Jr., J. P. May, and J. E. McClure, Ordinary RO(G)-graded cohomology, Bull. Amer. Math. Soc. (N.S.) 4 (1981), 208-212.

15. L. G. Lewis, Jr., J. P. May, and M. Steinberger (with contributions by J. E. McClure), Equivariant stable homotopy theory, Lecture Notes in Math., vol. 1213, Springer, 1986.

16. W. Lück, Transformation groups and algebraic K-theory, Lecture Notes in Math., vol. 1408, Springer, 1989.

17. S. Mac Lane, Categories for the working mathematician, Springer-Verlag, 1971.

18. T. Matumoto, Equivariant cohomology theories on G-CW complexes, Osaka J. Math. 10 (1973), 51-68.

19. U. Namboodiri, Equivariant vector fields on spheres, Trans. Amer. Math. Soc. 278 (1983), 431-460.

20. N. Popescu, Abelian categories with applications to rings and modules, London Math. Soc. Monographs, No. 3, Academic Press, 1973.

21. S. Waner, $G-C W(V)$ complexes, unpublished manuscript.

22. __ Mackey functors and G-cohomology, Proc. Amer. Math. Soc. 90 (1984), 641-648.

23. G. W. Whitehead, On spaces with vanishing low-dimensional homotopy groups, Proc. Nat. Acad. Sci. U.S.A. 34 (1948), 207-211.

Department of Mathematics, Syracuse University, Syracuse, New York 13244-1150 